\title{
Numerical studies of the phase diagram of layered type II superconductors in a magnetic field
}

\author{
A.K.Kienappel and M.A.Moore \\ Department of Physics, University of Manchester, Manchester, M13 9PL, United Kingdom.
}

(December 5, 2017)

\begin{abstract}
We report on simulations of layered superconductors using the Lawrence-Doniach model in the framework of the lowest Landau level approximation. We find a first order phase transition with a $B(T)$ dependence which agrees very well with the experimental "melting" line in $\mathrm{YBa}_{2} \mathrm{Cu}_{3} \mathrm{O}_{7-\delta}$. The transition is not associated with vortex lattice melting, but separates two vortex liquid states characterised by different degrees of short-range crystalline order and different length scales of correlations between vortices in different layers. The transition line ends at a critical end-point at low fields. We find the magnetization discontinuity and the location of the lower critical magnetic field to be in good agreement with experiments in $\mathrm{YBa}_{2} \mathrm{Cu}_{3} \mathrm{O}_{7-\delta}$. Length scales of order parameter correlations parallel and perpendicular to the magnetic field increase exponentially as $1 / T$ at low temperatures. The dominant relaxation time scales grow roughly exponentially with these correlation lengths. We find that the first order phase transition persists in the presence of weak random point disorder but can be suppressed entirely by strong disorder. No vortex glass or Bragg glass state is found in the presence of disorder. The consistency of our numerical results with various experimental features in $\mathrm{YBa}_{2} \mathrm{Cu}_{3} \mathrm{O}_{7-\delta}$, including the dependence on anisotropy, and the temperature dependence of the structure factor at the Bragg peaks in neutron scattering experiments is demonstrated.
\end{abstract}

PACS numbers: 74.20.De, 74.25.Dw, 74.25.Ha

\section{INTRODUCTION}

In the mean-field limit the phase diagram of type II superconductors has two phases: the normal state and the mixed state in which the lines of magnetic flux are arranged in a triangular Abrikosov lattice [1]. However, thermal fluctuations destroy the flux lattice near the mean-field transition line and a flux liquid phase enters the phase diagram [2]. As the temperature is reduced the vortex liquid undergoes a first order phase transition to what is commonly assumed to be the flux lattice state. This leads to a phase diagram as shown in Fig. 1(a). The strong belief in first order melting of the Abrikosov flux lattice rests on the experimental evidence reviewed in Sec. IA. Much of the analytical work on vortex lattice melting relies on the Lindemann criterion, which states that melting occurs if the mean fluctuation radius of a lattice point around its equilibrium position has reached a certain fraction (usually between 0.1 and 0.2 ) of the lattice constant [3]. This criterion is not rigorous and does not provide a satisfying thermodynamic melting theory. The possibility of a first order phase transition due to decoupling of the different layers has also been investigated [4. However, a decoupling transition is mostly expected to occur in addition to melting, and the lack of experimental evidence for two separate phase transitions has lead to a widespread belief that either there is no sharp decoupling transition or that it occurs simultaneously with flux lattice melting. Our numerical results suggest a phase diagram which is fundamentally different from Fig. 1 1(a). It has a first order phase transition in excellent agreement with the first order transition line in $\mathrm{YBa}_{2} \mathrm{Cu}_{3} \mathrm{O}_{7}$ (YBCO) in the $B-T$ plane (see Sec. III). However, this transition is a decoupling transition and not associated with vortex lattice melting. There is only one phase in the phase diagram: the vortex liquid phase, and a vortex lattice exists only at zero temperature.

Although there is striking experimental evidence for a first order phase transition in both $\mathrm{YBCO}$ and $\mathrm{Bi}_{2} \mathrm{Sr}_{2} \mathrm{CaCu}_{2} \mathrm{O}_{8}$ (BSCCO), there are certain features of the experimental data that are not explained by the standard vortex lattice melting picture, most importantly the loss of first order behavior along the transition line at high (both for $\mathrm{YBCO}$ and $\mathrm{BSCCO}$ ) and low (YBCO only) fields. Note that an end of the first order phase transition line at a critical end-point is not possible for a vortex lattice melting line, because the phase boundary separates phases of different symmetry. Our first order transition is not associated with any symmetry breaking. Thus the existence of a low field critical end-point should be expected and is directly observed in the simulation.

In the framework of a vortex lattice melting picture the disappearance of the first order melting line can be explained by the presence of a tricritical point where the first order transition changes to a continuous one. Such behavior is commonly assumed to occur as an effect of sample disorder, which is to a certain degree present even in the best crystals. However, there is no wide consensus on the phase diagram in the presence of disorder. The three most important categories to distinguish are the disordered liquid, vortex glass and Bragg glass scenarios. For the first case there is no phase which is ther- 
modynamically distinct from a vortex liquid and thus no thermodynamic phase transition. However, a fairly sharp crossover from fast to slow dynamics may occur within the vortex liquid state. The vortex glass scenario presented in detail in Ref. [5] relies on analogy to spin glass behavior. The vortex liquid is expected to freeze via a continuous transition to a vortex glass state characterized by short-range crystalline correlations but longrange phase correlations. Such a vortex glass phase would be truly superconducting with vanishing dc resistance. A popular recent theory predicts for weak disorder a first order transition to a Bragg glass state, which is characterized by slow, at most algebraic, decay of translational crystalline order [6].

(a)

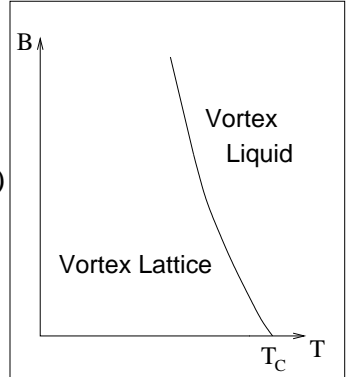

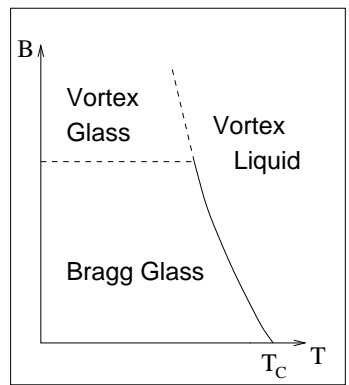

(b)

FIG. 1. Popular phase diagrams (a) in the clean case and (b) in the presence of disorder. Solid and dotted lines mark first order and continuous transitions respectively.

With this inconclusive theoretical background, experimental and numerical evidence have had a major impact on the picture of the phase diagram of high temperature superconductors (HTSC) including fluctuations and disorder. A popular phase diagram including disorder which accounts for many experimental features, notably the loss of first order behavior at high fields, is shown in Fig. Il(b). Our numerical results with disorder give a phase diagram with only one phase, a vortex liquid, just as in the clean limit.

\section{A. Experimental evidence}

This section attempts a review of experimental evidence on which both our and more conventional pictures of the phase diagram of layered superconductors are based. We discuss only evidence in YBCO, because this is the material to which our numerical model applies naturally.

\section{First order transition}

There is striking experimental evidence for a first order transition in YBCO. The earlier evidence for discontinuous behavior suggesting a first order transition came from resistive measurements [7]. A sharp drop in resistivity was found to occur at a temperature well below the $H_{c 2}$ line. Later it was shown that these resistive drops coincide with a discontinuity in the magnetization, the first thermodynamic quantity found to be discontinuous at the transition line [8,9]. The first measurements of the latent heat which unambiguously characterises a first order transition, were made by Schilling et al. 10 in 1996. Since then a latent heat at the first order vortex transition has been observed in different crystals of $\mathrm{YBa}_{2} \mathrm{Cu}_{3} \mathrm{O}_{7-\delta}$ with varying oxygen deficiencies $\delta[11,12$ and for different orientations of the applied field [13]. The $B(T)$ dependence of the first order transition line obeys the standard continuum anisotropic scaling rules [14] under rotation of the applied field away from the $c$-axis [13].

The scaling behavior of the first order transition lines for samples with different oxygen deficiencies $\delta$ and therefore different mass anisotropies $\gamma$ is of some interest as it can be easily compared to predictions of different theoretical models. In a range of samples the first order lines have been found to scale with $1 / \gamma$ by Roulin et al. [12], i.e. $\gamma B(T)$ collapses on one scaling curve. This is in disagreement with standard London-Lindemann type vortex lattice melting theory, which predicts the melting curve to scale inversly with the Ginsburg number $G i$ as $1 / G i \propto 1 / \gamma^{2}[3]$. The $1 / \gamma$ scaling form is consistent with 3D lowest Landau level (LLL) scaling and our numerical results.

\section{Loss of first order behavior}

The first order behavior at the vortex transition has been observed to vanish at an upper critical field $B_{u c}$ for samples which are not fully oxygenated [12]. $B_{u c}$ is found to increase with decreasing concentration of oxygen deficiencies $\delta$ in $\mathrm{YBa}_{2} \mathrm{Cu}_{3} \mathrm{O}_{7-\delta}$. For $\delta=0$ a latent heat can be observed up to the highest experimentally investigated fields of $16 \mathrm{~T}$ 11]. The end of the first order line thus appears to be strongly correlated with the amount of point disorder in the form of oxygen vacancies present in the system. The upwards shift of $B_{u c}$ with increasing oxygen content fits in well with the theoretical phase diagram in Fig. 1. (b), where it corresponds to an extension of the Bragg glass phase to higher fields with decreasing disorder.

Another striking feature of the experimental first order transition line in YBCO is its termination at low magnetic fields, which has been consistently observed in all relevant calorimetric measurements 10 13]. The latent heat disappears for fields smaller than some lower critical field $B_{l c}$. The existence of a low field end-point is usually found "puzzling" [12]. The variations of $B_{l c}$ for specific heat measurements in different samples are large and qualitatively unexplained in the framework of 
a vortex lattice melting picture. For $B \| c$ in near optimally doped samples with a high level of oxygen deficiency $\delta>0.06, B_{l c}$ is approximately 0.7T [10]. Measurements of $B_{l c}$ in different samples show that $B_{l c}$ increases with oxygen content [12], which suggests at first sight a correlation with twin density. The value of $B_{l c}$ in fully oxygenated, twinned samples is of the order of several Tesla [11]. The authors of Ref. [12] discuss the origin of the end-point and the variation of $B_{l c}$ in different samples. The fact that detwinning does not noticeably change $B_{l c}$ and the existence of $B_{l c}$ in naturally untwinned samples, together with the reproducibility of $B_{l c}$ in different fully oxygenated samples, leads them to the conclusion that an intrinsic mechanism as a cause for the end point cannot be excluded.

An important relation for our discussion of the value of $B_{l c}$ in different YBCO samples (see Sec. III C) is that an increase in oxygen content corresponds not only to an increase in natural twin density, but also to a systematic decrease in the anisotropy $\gamma$ in the samples used in Ref. [12,15. Our work suggests that this change in anisotropy rather than the presence of twins may cause the change in $B_{l c}$. Our numerical work provides an explanation for the existence of $B_{l c}$ as well as a qualitatively correct prediction of its rapid increase when $\gamma$ is decreased, such as can be achieved by increasing the oxygen content. Another noteworthy point which we shall discuss in Sec. IV B2 is that the location of $B_{l c}$ according to magnetization measurements is not always in agreement with the one measured in specific heat measurements. In a fully oxygenated sample in Ref. 11 the latent heat vanishes at ca. $6 \mathrm{~T}$ while a magnetization discontinuity is still observed down to a field of $4 \mathrm{~T}$.

Transport measurements reflect the loss of first order character of the transition for low as well as for high fields [7,16]. The resistance only drops to zero, which would be the expected resistance for a weakly pinned lattice at the very low voltages used, for a limited range of magnetic fields. For high and low fields only a fractional drop is visible, which disappears completely somewhat below 2 and above 7 Tesla.

Below $B_{l c}$ and above $B_{u c}$ as well as in samples where no latent heat at all is observed, a "step" in the heat capacity $C$ remains 1012,15 . This behavior has been interpreted as evidence for a second order transition. The sharpness of this "step", is not altogether convincing (see e.g. Fig. 3 in Ref. 13). However, the existence of a continuous transition to a vortex glass state at high fields is expected for the theoretical phase diagram in Fig. 11(b). According to the same phase diagram another line marking a field driven phase transition line from Bragg glass to vortex glass is expected to emerge where first order melting turns continuous [6]. The "fishtail" magnetization anomaly 47, which correlates with the location of $B_{u c}$ [17] could be interpreted as evidence for such a transition. A lack of sharpness of this feature makes it a candidate, however, for a crossover rather than a phase transition. There has also been evidence from resistive measurements for a field driven crossover line as an extension of the first order transition in YBCO [18].

\section{Evidence for a vortex lattice}

A vital ingredient of the vortex lattice melting scenario which this paper disputes is the existence of a vortex lattice. Experimentally a vortex lattice is indistinguishable from a liquid or glassy phase with short-range crystalline order on length scales large compared to the vortex separation. Evidence for hexagonal coordination over large distances can be seen in YBCO for low fields in Bitter pattern decoration experiments 19. At high fields this technique fails because the vortices are too close to be individually resolved. A powerful method to detect longrange vortex correlations is neutron scattering [20]. The Bragg peaks observed in these experiments show that vortex positions are long-range correlated in all directions. The correlation length along the field can be enhanced by twin boundaries if the field is oriented along the $c$-axis. However, data from experiments with different orientation of the applied magnetic field show similar results, and thus indicate that the long correlation lengths along the field are independent of the presence of twin planes. The intrinsic crystalline in-plane correlation length is more difficult to deduce from neutron scattering data, because twin boundaries and/or pinning to the underlying crystal determine preferred orientations and can thereby strongly enhance orientational order 21.

Although neutron scattering experiments give evidence for long-range vortex correlations, some features of the data are unexpected in the framework of a vortex lattice melting picture. The observed diffraction patterns suggest the existence of a vortex lattice or a Bragg glass, which means that the melting transition is expected to be of first order. Such a first order melting transition should be visible as a discontinuous appearance of Bragg peak intensity, as the temperature is reduced. However, the peaks appear continuously, which leads the authors of Ref. [20] to the conclusion that they must be dealing with second order vortex glass melting.

An additional feature of the melting line defined by the onset of Bragg peaks (which is not mentioned in Ref. [20]) is that it lies in the $B-T$ phase diagram distinctly below the line corresponding to thermodynamically measured first order transition lines 10,12 under the assumption of scaling with mass anisotropy like $B \propto 1 / \gamma$ or $B \propto 1 / \gamma^{2}$. This point will be investigated in more detail in Sec. IV A 2. For the alternative phase diagram presented in this paper, a continuous onset of Bragg peak intensity somewhat below the first order transition line is just the expected behavior. 


\section{B. Numerical simulations}

A large number of numerical Monte Carlo or Langevin dynamics simulations of different three dimensional models have with few exceptions provided evidence of first order vortex lattice melting. The main disagreement between different simulations of layered models concerns the question whether layer decoupling coincides with this melting transition. In this section we give an overview of different numerical results and point out what we consider to be the weaknesses of the respective models used.

From the frustrated XY and Villain and lattice London models there is evidence for first order melting and distinct decoupling 22 as well as, at least in the thermodynamic limit, only one simultaneous first order melting and decoupling transition [23]. Very recent simulations of the uniformly frustrated 3D XY model show a vortex lattice melting transition as well as a second, possibly first order, phase transition within the liquid phase, at which the vortex line tension goes to zero 24. In these models vortices are confined to a lattice. In $3 \mathrm{D}$, a lattice acts as a close grid of columnar pins with infinite pinning potential in the thermodynamic limit which may lead to spurious phase transitions. For the LLL, alteration of the phase diagram by the presence of a lattice pinning potential which breaks translational and rotational invariance has been predicted from a theoretical analysis [25].

There are also numerical models that avoid using a lattice. Numerical models relying on the 2D Bose gas analogy yield simultaneous melting and loss of phase coherence along the $c$-axis [26]. A different scenario has been seen in a simulation by Wilkin and Jensen [27], in which vortex pancakes in different layers are represented by particles with in-layer short-range repulsive and interlayer attractive interactions. A first order transition associated with decoupling of vortices and without melting character is observed. At a lower temperature a melting crossover without noticeable thermodynamic signature occurs. When sufficient point disorder is added, the decoupling transition loses its first order character. While not being affected by pinning to a numerical lattice, the latter models may give unrealistic results because they allow variation of vortex position only, neglecting fluctuations of order parameter magnitude and in many cases having unrealistic short-range interactions [28]. Simulations using the Lawrence-Doniach (LD) model in the LLL limit, which allows for these fluctuations and which has long-range vortex interactions, show a single first order simultaneous melting and decoupling transition [29,30]. All of the simulations mentioned in this paragraph use periodic boundary conditions perpendicular to the field, which we believe can also lead to unphysical results (see Sec. II).

In the following sections we introduce our numerical model (Sec. III) and report results from our simulation.
Comparisons to experimental data on YBCO are made in each section in the context of the relevant numerical results. Sec. III addresses the numerical phase diagram of layered systems in the clean limit, followed by an analysis of order parameter correlations in space and time in Sec. IV. Numerical results in the presence of quenched random disorder are reported in Sec. V. The paper closes with a discussion and a summary of our work.

\section{NUMERICAL MODEL}

Our simulation of a layered superconductor uses the Lawernce-Doniach (LD) model [31], which consists of a stack of planes with Josephson coupling between neighboring layers. With the superconducting order parameter in the $n^{t h}$ layer denoted as $\psi_{n}$, the Hamiltonian for the layered system in a magnetic field perpendicular to the layers is

$$
\begin{aligned}
\mathcal{H}_{\text {clean }}= & \sum_{\mathrm{n}} d_{0} \int d^{2} r\left(\alpha\left|\psi_{n}\right|^{2}+\frac{\beta_{2 D}}{2}\left|\psi_{n}\right|^{4}+\right. \\
& \left.\frac{1}{2 m_{a b}}\left|(-i \hbar \nabla-2 e \mathbf{A}) \psi_{n}\right|^{2}+J\left|\psi_{n+1}-\psi_{n}\right|^{2}\right),
\end{aligned}
$$

where $d$ is the layer periodicity, $d_{0}$ is the layer thickness and $\mathbf{B}=\nabla \times \mathbf{A}$, which we shall take as constant and uniform. The same Hamiltonian can be read as the finite difference approximation to an anisotropic continuum model with $\psi(n d)=\sqrt{d / d_{0}} \psi_{n}, \beta=\beta_{2 D} \times d / d_{0}$ and $m_{c}=\hbar^{2} / 2 J d^{2}$. In first approximation $\alpha(T)=\alpha^{\prime}\left(T-T_{c}\right)$ and $\beta_{2 D}(T)$ is constant; $\alpha^{\prime}, \beta_{2 D}, J>0$.

We simulate the LD model with $N_{a b}$ vortices per layer in $N_{c}$ layers. Along the $c$-axis we use periodic boundary conditions. In the $a b$-planes we chose a different, more unusual approximation to the thermodynamic limit of an infinite plane. The layers are taken to be of spherical geometry with a radial magnetic field. The reasons for our preference of this geometry to the more widely used geometry of a plane with periodic boundary conditions have been discussed in detail by Dodgson and Moore [32]. The main advantage is that the spherical geometry guarantees full rotational and translational symmetry, which periodic boundary conditions do not. One example where the spherical geometry captures the physics better than periodic boundary conditions - despite the topological defects imposed on the triangular lattice ground state on the sphere [32] - are particles interacting with the $1 / r^{12}$ interaction. Here simulations on a sphere show already for moderate system sizes the genuine continuous transition to the crystalline state [33], while with periodic boundary conditions a spurious first order transition occurs even for very large system sizes.

For each layer $\psi$ is expanded in eigenstates of the squared momentum operator $(-i \hbar \nabla-2 e \mathbf{A})^{2}$. We retain eigenstates belonging only to the lowest eigenvalue 
$2 e B \hbar$, (the LLL approximation) which is a useful procedure over a large portion of the vortex liquid regime [34]. Our numerical model is an extension to the model of a spherical thin film used in Ref. 32, 35, 36]. The magnetic potential is $\mathbf{A}=B R \tan (\theta / 2) \hat{\phi}$, for which an orthonormal basis of the LLL eigenfunctions in each layer is given by

$$
\phi_{m}=\frac{A_{m}}{\left(4 \pi R^{2}\right)^{1 / 2}} e^{i m \phi} \sin ^{m}(\theta / 2) \cos ^{N_{a b}-m}(\theta / 2),
$$

where $A_{m}=\left(\left(N_{a b}+1\right) ! /\left(N_{a b}-m\right) ! m !\right)^{1 / 2}$ for $0 \leq m \leq$ $N_{a b}$. Note that we use units of length $l_{m}=(\hbar / 2 e B)^{1 / 2}$, which fixes the sphere radius as $R=\left(N_{a b} / 2\right)^{1 / 2}$.

The order parameter in every layer is expanded in the above basis set as

$$
\psi_{n}(\theta, \phi)=Q \sum_{m=0}^{N} v_{n, m} \phi_{m}(\theta, \phi)
$$

where $Q=\left(2 \pi k_{B} T / \beta_{2 D} d_{0}\right)^{1 / 4}$. Orthonormality of the LLL eigenfunctions can now be used to express the Hamiltonian in terms of the LLL coefficients $v_{n, m}$ and only two parameters, $\alpha_{2 T}$ and $\eta$ :

$$
\begin{aligned}
\frac{\mathcal{H}_{\text {clean }}}{k_{B} T}= & \sum_{n=1}^{N_{c}}\left(\alpha_{2 T} \sum_{m=0}^{N_{a b}}\left|v_{n, m}\right|^{2}+\frac{1}{2 N_{a b}} \sum_{p=0}^{2 N_{a b}}\left|U_{n, p}\right|^{2}+\right. \\
& \left.\left|\alpha_{2 T} \eta\right| \sum_{m=0}^{N_{a b}}\left|v_{n+1, m}-v_{n, m}\right|^{2}\right)
\end{aligned}
$$

with $U_{n, p}\left(\left\{v_{n, m}\right\}\right)$ as defined in Sec. A 1. The $2 \mathrm{D}$ effective temperature and field parameter for each layer is $\alpha_{2 T}=$ $\left(d_{0} h / 2 e \beta_{2 D} B k_{B} T\right)^{1 / 2} \alpha_{H}$, with $\alpha_{H}=\alpha(T)+e B \hbar / m_{a b}$. The scaling parameter $\eta$ relates to the Josephson coupling constant $J$ as $\eta=J /\left|\alpha_{H}\right|$. We can define an effective mass $m_{c}$ via $\eta=\hbar^{2} / 2 m_{c} d^{2}\left|\alpha_{H}\right|$. Then $\sqrt{\eta}$ is the ratio of the $3 \mathrm{D}$ mean-field coherence length to the layer periodicity, $\sqrt{\eta}=\xi_{\|} / d$. Note that for a HTSC material the $2 \mathrm{D}$ parameters $\beta_{2 D}$ and $d_{0}$ are effective microscopic properties of the copper oxide layers and essentially unknown. However, they enter the simulation only via $\alpha_{2 T}=\left(2 \pi d_{0} / \beta_{2 D} k_{B} T\right)^{1 / 2} \alpha_{H}$ where they can be replaced by the layer periodicity $d$ and the bulk $\beta$ using the relation $\beta=d / d_{0} \times \beta_{2 D}$.

The state in the LLL-LD model depends on two dimensionless scaling parameters, $\alpha_{2 T}$ and $\eta$. It is useful to have two scaling parameters which can be thought of as something physical, e.g. one characterising temperature and the other coupling strength between layers. In this sense, $\alpha_{2 T}$ and $\eta$ are not very appropriate. Because $d_{0}<d$, the temperature parameter $\alpha_{2 T}$ goes to zero independently of $B$ and $T$ in the $d \rightarrow 0$ limit of a continuous system. The coupling parameter $\eta$ includes a factor $\left|1 / \alpha_{H}\right|$, which means that it diverges at the mean-field
$H_{c 2}$. For these reasons we choose as effective temperature and coupling strength two different parameters that depend on $\alpha_{2 T}$ and $\eta$.

For the temperature parameter in a layered model describing a bulk sample, the 3D version of the LLL scaling variable $\alpha_{T}$ [2] stands out as an appropriate candidate. It is given by

$\alpha_{T}=\left(\frac{\sqrt{2} \hbar^{3 / 2} \pi}{k_{B} e^{3 / 2} \mu_{0}}\right)^{2 / 3}\left(\frac{1}{\kappa^{2} \gamma}\right)^{2 / 3} \frac{\partial B_{c 2} /\left.\partial T\right|_{T_{c}}\left(T-T_{c}\right)+B}{(B T)^{2 / 3}}$,

which can be expressed in terms of $\alpha_{2 T}$ and $\eta$ as $\left|\alpha_{T}\right|^{3}=$ $\eta\left(2 \alpha_{2 T}\right)^{4}$. At low temperatures $\left|\alpha_{T}\right|^{3 / 2}$ behaves as $1 / \mathrm{T}$. A good measure of coupling strength is the product $\left|\alpha_{2 T} \eta\right|$ which multiplies the coupling term in the Hamiltonian. It is in SI units given by

$$
\left|\alpha_{2 T} \eta\right|=\left(\frac{\hbar^{3} \pi}{8 e^{3} k_{B} \mu_{0}}\right)^{1 / 2} \frac{1}{\kappa \gamma^{2} d^{3 / 2}} \frac{1}{(B T)^{1 / 2}} .
$$

Other than the factor $1 / \sqrt{B T},\left|\alpha_{2 T} \eta\right|$ contains only constants and therefore varies slowly for rather a wide range of $\alpha_{H}$. It can thus be regarded as a material constant over considerable regions of the $B-T$ plane. The limiting case $\left|\alpha_{2 T} \eta\right| \rightarrow 0$ describes the $2 \mathrm{D}$ system, while small $\left|\alpha_{2 T} \eta\right|$ means strongly layered characteristics. For constant $\alpha_{T}$, the limit $\left|\alpha_{2 T} \eta\right| \rightarrow \infty$ is the continuum limit, in which all system properties depend on $\alpha_{T}$ alone. Note that our model parameters depend on the bulk material parameters $\kappa$, mass anisotropy $\gamma$, layer separation $d$, $\partial B_{c 2} /\left.\partial T\right|_{T=T_{c}}, T_{c}$ as well as $B$ and $T$ only. All of these are for HTSC more or less well known from experiment.

Most of the experimental evidence to which we can compare our results is from samples which have at least weak disorder due to crystal defects or impurities. An idealized version of this kind of disorder is a random local variation of $T_{c}$. To simulate the effects of such quenched random disorder, a random local potential $\Theta$ can be added to the quadratic energy term of every layer, to give a disorder contribution to the Hamiltonian of

$$
\mathcal{H}_{\text {dis }}=d_{0} \int d^{2} r \Theta(\mathbf{r})|\psi(\mathbf{r})|^{2}
$$

where $\Theta(\mathbf{r})$ is real and Gaussian distributed with

$$
\begin{gathered}
\overline{\Theta(\mathbf{r})}=0 \\
\overline{\Theta(\mathbf{r}) \Theta\left(\mathbf{r}^{\prime}\right)}=\Delta \delta\left(\mathbf{r}-\mathbf{r}^{\prime}\right) .
\end{gathered}
$$

Here $\delta$ is the two dimensional Dirac delta function and $\Delta$ is the measure of the strength of the disorder. The random realizations of $\Theta$ are different in every layer, and $\mathcal{H}_{\text {dis }}$ is the sum of the single layer contributions. The disorder contribution $\mathcal{H}_{\text {dis }}$ in terms of the LLL coefficients as used in our simulation is given in the Appendix, Sec. 
A 2. Unfortunately there is no experimental measure of $\Delta$, and we can therefore not express the strength of disorder in terms of measurable quantities.

As in reference [35], our simulation follows Langevin (model A) dynamics. We drive our system by the time dependent Ginsburg-Landau equation, discretized in time and expanded in the appropriate eigenfunctions:

$$
v_{n, m}(t+\Delta t)-v_{n, m}(t)=-\Delta t \Gamma \frac{\partial \mathcal{H}(t)}{\partial v_{n, m}^{*}}+\Delta t \xi_{n, m}(t) .
$$

The complex random noise variables $\xi_{n, m}$ are drawn independently from a Gaussian probability distribution, so that their magnitude has a variance $\sigma^{2} / \Delta t=1$, where $\sigma^{2}=2 \Gamma k_{B} T$, so that $\Delta t$ is the only free parameter. We chose $\Delta t=0.15$ (see Ref. [35]).

\section{NUMERICAL PHASE DIAGRAM}

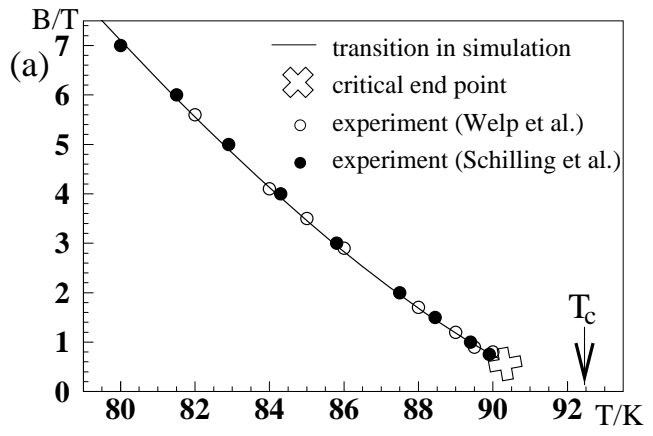

$\Delta(4 \pi \mathrm{M} / \mathrm{G})$

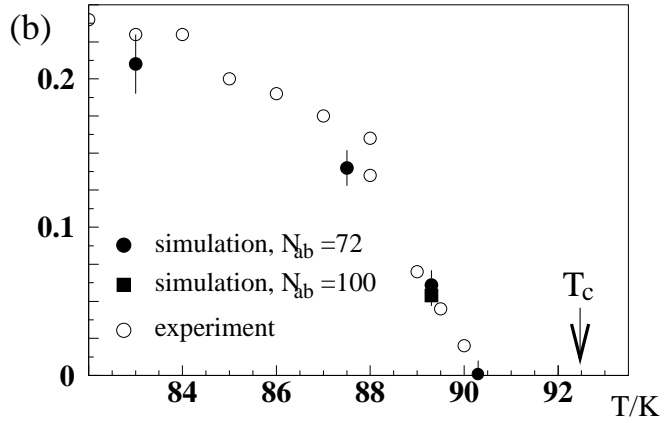

FIG. 2. Phase diagram (a) and magnetization discontinuity (b). Experimental data is taken from Ref. [8,10] (a) and [10] (b). (For $N_{c}$ see section III B)

As an introduction to our numerical results we show in Fig. 2(a) our numerical phase diagram for the clean case in comparison to experimental results. The only phase present in this phase diagram is a vortex liquid. We see a first order transition line between two vortex liquid states with a critical end-point at low fields, which agrees well with the experimental YBCO "melting" line. The magnetization jumps we observe are shown in Fig. 2(b). They are in very good agreement with data for $\mathrm{YBCO}$ from
Ref. [10]. The magnetization jumps in these experimental measurements are very likely to be of thermodynamic origin, as they are found to be consistent (according to the Clausius Clapeyron relation) with the latent heat data measured in the same sample [10]. Figure 2(a) and (b) were obtained using standard YBCO values for the fitting parameters; viz for the Landau-Ginsburg parameter $\kappa=60$, the mass anisotropy $\gamma=7.5$, the slope of the mean-field transition line $\partial B_{c 2} /\left.\partial T\right|_{T=T_{c}}=-2 \mathrm{~T} / \mathrm{K}$, the mean-field $T_{c}=92.5 \mathrm{~K}$ and the layer separation $d=11.4 \AA$.

\section{A. Continuum limit}

From LLL scaling [2] we know that all thermodynamic properties depend on $\alpha_{2 T}$ alone in the 2D limit $\left(\left|\alpha_{2 T} \eta\right| \rightarrow 0\right)$ and on $\alpha_{T}$ alone in the continuum limit $\left(\left|\alpha_{2 T} \eta\right| \rightarrow \infty\right)$. Figure 3 shows how the thermal average of a typical quantity of interest, here the Abrikosov ratio $\beta_{A}=\left\langle|\psi|^{4}\right\rangle /\left\langle|\psi|^{2}\right\rangle^{2}$, behaves in the intermediate regime of a positive finite coupling strength.
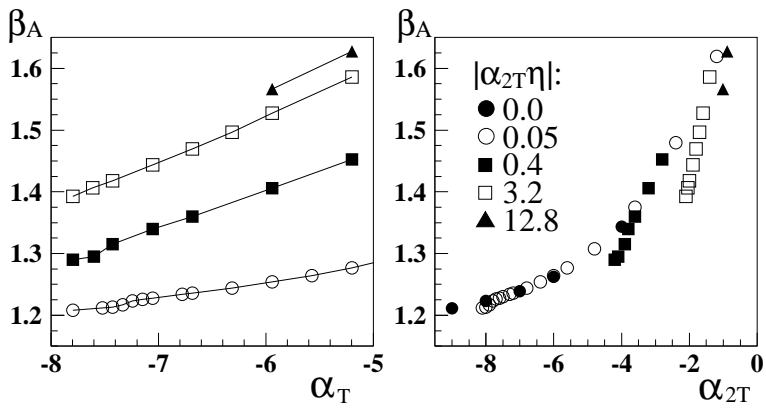

FIG. 3. Abrikosov ratio $\beta_{A}$ for a range of coupling strengths $\left|\alpha_{2 T} \eta\right|$ plotted against the 3D and 2D effective temperature variables $\alpha_{T}$ and $\alpha_{2 T}$. Solid lines are guide to the eye.

In the high temperature regime 2D scaling applies, in the low temperature regime however 3D scaling becomes more appropriate. If we look at our model as a finite difference approximation to the continuum case, this means that this approximation is for the same layer spacing $d / \xi_{\|}=1 / \sqrt{\eta}$ better at low than at high $3 \mathrm{D}$ temperatures $\alpha_{T}$. This is a natural result as correlations along the field direction increase in the layered system as $\alpha_{T}$ decreases. We are, however, not able to simulate system sizes that behave fully continuum-like at moderate temperatures. The numbers of layers used for the data in Fig. 3 are chosen to have the correlations along the $c$ axis distinctly smaller than the system size, which means for $\left|\alpha_{2 T} \eta\right|=12.8$ even at the moderate temperature of $\alpha_{T}=-6$ an $N_{c}$ of 200 . 


\section{B. First order transition}

Figure $4(a)$ shows the phase diagram in terms of simulation parameters, where data points mark the location of first order transition points as found upon heating and cooling the system. The logarithmic scale is chosen for even data distribution. As $\left|\alpha_{2 T} \eta\right|$ increases and the system approaches the continuum limit, the transition line terminates at a critical end-point. Note that along the transition line $\alpha_{T}$ is approximately constant, which means that the field and temperature dependence of the transition line behave as expected for a continuum model where $\alpha_{T}$ is the only scaling parameter in the system. Another point of interest is the limit $\left|\alpha_{2 T} \eta\right| \rightarrow 0$ of independent 2D layers. If we approximate the transition value of $\alpha_{T}$ as constant for different $\left|\alpha_{2 T} \eta\right|$, this translates to a dependence of $\alpha_{2 T}$ on $\left|\alpha_{2 T} \eta\right|$ along the transition line as $\alpha_{2 T} \propto-\left|\alpha_{2 T} \eta\right|^{-1 / 3}$. As the coupling is reduced to zero, the $2 \mathrm{D}$ transition temperature diverges, $\alpha_{2 T} \rightarrow-\infty$. This would imply that there is no finite temperature phase transition in two dimensions, in agreement with 2D simulation results [32,36, 35]. Such behavior would be a natural for a transition of a predominantly decoupling nature, which cannot occur in thin films. However, we find that direct extension of our numerical transition line to even lower couplings and thereby numerical observation of the 2D limiting behavior is impossible due to the finite size effects analyzed in Appendix $\mathrm{C}$.

Figures 1 (b)-(d) show examples of the kind of measurement used to locate the first order transition. The system displays hysteresis upon heating and cooling. We mark the transition in the middle of the observed hysteresis loop. The hysteresis decreases with sweeping rate (typically 10,000-20,000 time steps per data point), and for a few cases we have confirmed with equilibrium measurements that the equilibrium transition coincides roughly with the middle of the hysteresis loop. The coupling values for which we show hysteresis measurements correspond in decreasing order firstly to the critical end-point, secondly the nearest to the critical end-point where we have measured hysteresis, and thirdly an arbitrary, low coupling value. The discontinuity $\Delta \rho$ in the order parameter density $\rho$, given by $\rho=\left(\alpha_{T} \beta / 2 \pi \alpha_{H}\right) \times\left\langle|\psi|^{2}\right\rangle$, is found to be more or less constant between $\left|\alpha_{2 T} \eta\right|=0.1$ and 1.5. A decrease in $\Delta \rho$ is observed below $\left|\alpha_{2 T} \eta\right|=0.1$. However, this decrease at low couplings is possibly due to finite size effects, which are in detail described in the Appendix 9. The rapid decrease to zero between $\left|\alpha_{2 T} \eta\right|=1.5$ and $\left|\alpha_{2 T} \eta\right|=2.5$ appears system size independent.

The plots of the hysteresis in the degree of independence of neighboring layers, given by

$$
\Gamma=\frac{\left\langle|\psi(\mathbf{r})-\psi(\mathbf{r}+d \hat{c})|^{2}\right\rangle}{\left\langle|\psi|^{2}\right\rangle}
$$

reveal more about the nature of the transition and its disappearing. For low coupling, there is a large jump in $\Gamma$ at the transition, which decreases throughout parameter space until it is very small just before the end-point. The decoupling character of the transition gradually decreases along the transition line until it disappears at the critical end-point. Further discussion predicting the existence and approximate location of the critical end-point from the decoupling character of the transition and more detailed numerical results concerning the critical end-point will be given in Sec. III . The reader should note that in our simulation the onset of decoupling does not imply the onset of a superfluid density at the transition.
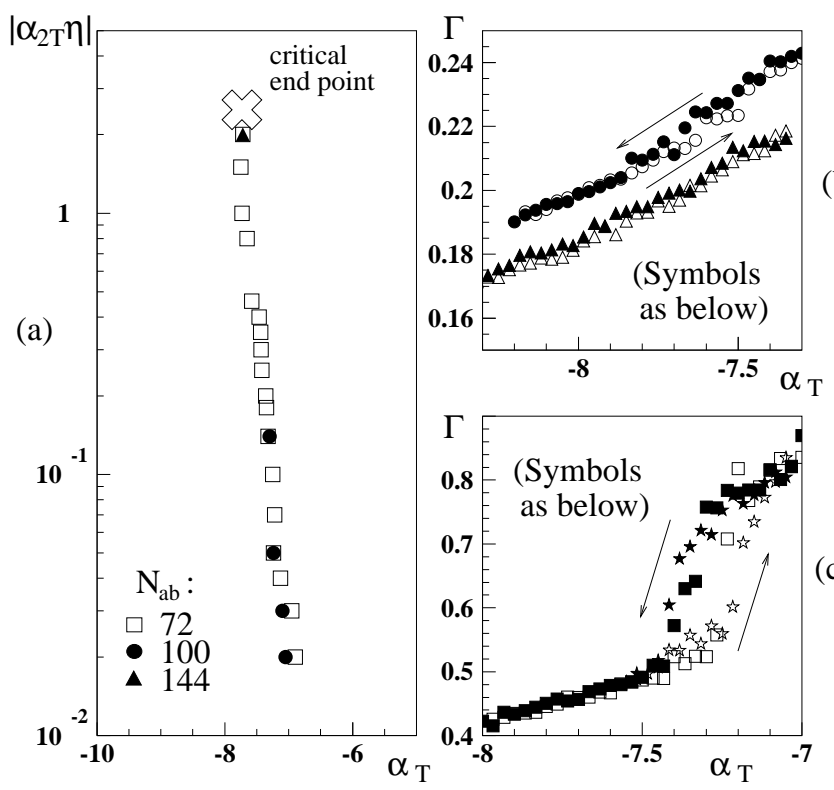

(b)

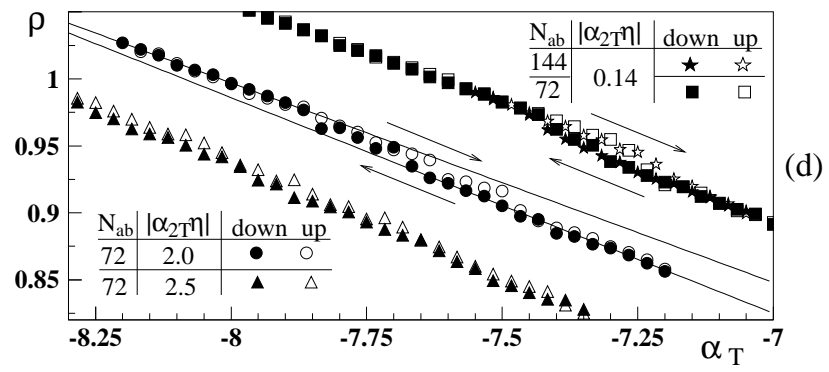

FIG. 4. Plot (a) shows the numerical phase diagram. First order transition points are plotted in the $\alpha_{T}-\left|\alpha_{2 T} \eta\right|$ plane. Plots (b)-(d) show order parameter density $\rho$ and the degree of layer independence $\Gamma$ upon heating and cooling. Note the hysteresis in the system, the clear first order behavior for $\left|\alpha_{2 T} \eta\right|=2$ (solid lines are linear fits of $\rho$ above and below the transition) and the lack of first order behavior for $\left|\alpha_{2 T} \eta\right|=2.5$. $N_{c}$ varies between 8 and 80 for $\left|\alpha_{2 T} \eta\right|$ between 0.02 and 2.5. For $\left|\alpha_{2 T} \eta\right|=2.5, \rho$ is offset by -0.05 .

The magnetization discontinuities in Fig. 2(b) are calculated from the discontinuities in $\rho$. The magnetiza- 
tion in the LLL model is $4 \pi M=-\left(\mu_{0} e \hbar / m_{a b}\right)\left\langle|\psi|^{2}\right\rangle$, which is in terms of our simulation parameters $4 \pi M=$ $\pi\left(B-B_{c 2}(T)\right) \rho / \alpha_{T} \kappa^{2}$, where $B$ is the applied magnetic field. Thus we can work out the magnetization discontinuity from the discontinuity in $\rho$ taken from the two linear extrapolations at the transition. The data points in Fig. 2(b) represent $\left|\alpha_{2 T} \eta\right|=1,1.5,2$ and 2.5 for $N_{c}=50$, $60,60 / 80,80$. For these four transition points we have an average value of $\alpha_{T}=-7.72$, which yields the transition line in Fig. 2.

The magnetization discontinuity is thermodynamically linked to the entropy change at the transition by the Clausius-Clapeyron relation

$$
\Delta S=-\Delta M \frac{d H_{F O T}}{d T},
$$

where $d H_{F O T} / d T$ is the slope of the first order transition line. We cannot measure $\Delta S$ directly. However, the good agreement in $\Delta M$ and the first order transition line between simulation and experiment on the one hand and the consistency of the experimental $\Delta S$ as calculated from magnetization and latent measurements for the samples in Ref. [10] on the other hand imply agreement for $\Delta S$ between simulation and experiment. Note also the clear change in slope of the linear fits to $\rho$ at the transition. Locally $\alpha_{T}$ is linear in $T$, and via Maxwell's relation

$$
\left.\frac{\partial S}{\partial H}\right|_{T}=\left.\frac{\partial M}{\partial T}\right|_{H}
$$

the sudden change in slope of $\rho$ at the transition implies a change in slope of the entropy and a step-like feature in the specific heat $C=T(\partial S / \partial T)_{B}$, with a lower value on the low temperature side of the transition. This is consistent with experiment. The relative change in slope we find from the fits in Fig. 1 is $8 \%$, while the equivalent experimental change in the heat capacity as taken from Fig. 3 of reference [13] is of the order of $5 \%$ (for a derivation of this value see Sec. IIIE). The fact that our simulation gives evidence for a step in the specific heat is consistent with results from a theoretical analysis showing that the step seen in experiment can be accounted for by thermal fluctuations within the LLL approximation [37].

\section{The critical end-point}

Until now we have as evidence for a critical end-point only the fact that the hysteresis along the transition line eventually becomes unobservable. To more firmly establish its existence, we shall consider how the nature of the transition may lead to a critical end-point.

\section{Why does the first order transition disappear?}

A well known first order phase transition which ends at a critical end-point is that of the ordinary liquid-gas transition. Here the phase transition separates a liquid state with small interparticle separation $d_{l}$ which takes advantage of the attractive interparticle energy which exist at distances $d_{m i n}$, so $d_{l} \approx d_{m i n}$, from a gaseous state with large interparticle separations $d_{g}$ which is favored by a high entropy. If the density is increased to the point where it reduces $d_{g}$ to be of order $d_{m i n}$, the transition line ends. We believe that in our case the entropy advantage of the high temperature phase arises when the order parameter values in adjacent layers are uncorrelated i.e when the layers are decoupled. The ratio of the meanfield coherence length perpendicular to the layers to the layer distance, $\xi_{\|} / d=\sqrt{\eta}$, increases along the transition line with increasing coupling parameter $\left|\alpha_{2 T} \eta\right|$. Because $\xi_{\|}$defines the minimal extent of order parameter correlations, a high-entropy state with decoupled layers is not possible if $\xi_{\|} \geq d$. And indeed, we will in the next section locate the critical end-point where the transition disappears at $\left|\alpha_{2 T} \eta\right|=2.55$ and $\alpha_{T}=-7.75$, which corresponds to $\sqrt{\eta}=1.06$. Very near the zero-field transition temperature $T_{c}$, where $\xi_{\|} \gg d$, the system can be expected to behave like a continuum and thus a decoupling line cannot be expected to reach $T_{c}$.

\section{Divergence of length scales}

Near a critical end-point we do not only expect all discontinuities to disappear, but we also expect there to be a divergence of the length scale of fluctuations in the order parameter density of the system. We therefore looked at the density-density correlations of the order parameter,

$$
\begin{aligned}
& C_{d}\left(\mathbf{r}^{\prime}, t^{\prime}\right)= \\
& \frac{\left\langle|\psi(\mathbf{r}, t)|^{2}\left|\psi\left(\mathbf{r}+\mathbf{r}^{\prime}, t+t^{\prime}\right)\right|^{2}\right\rangle-\left\langle|\psi(\mathbf{r})|^{2}\right\rangle\left\langle\left|\psi\left(\mathbf{r}+\mathbf{r}^{\prime}\right)\right|^{2}\right\rangle}{\left\langle|\psi|^{2}\right\rangle^{2}}
\end{aligned}
$$

in the case where $\mathbf{r}^{\prime}$ is a vector parallel to the $c$-axis and $t^{\prime}=0$. This correlator is expressed in terms of thermal averages of the LLL coefficients in the appendix, Sec. B2. Plots of these correlations near the critical end-point can be seen in Fig. 5 .

There is evidence of two length scales in the vicinity of the end-point. The short distance decay of the correlation function is dominated by the positional correlations of the vortices in the different layers. This length scale is mostly determined by $\alpha_{T}$ and changes slowly in the vicinity of the critical end-point. The diverging length scale is thus not that of positional correlations of the vortices, but instead is associated with local density fluctuations. This is not surprising if the analogy of an 
ordinary liquid-gas transition is considered, where crystalline correlations on a microscopic scale correspond to positional vortex correlations. A noteworthy feature of the short distance decay of vortex correlations is that the difference between curves at $\alpha_{T}=-7.6$ and $\alpha_{T}=-7.8$ decreases as the coupling parameter $\left|\alpha_{2 T} \eta\right|$ is raised past the end-point, as one would expect for a disappearing discontinuity.

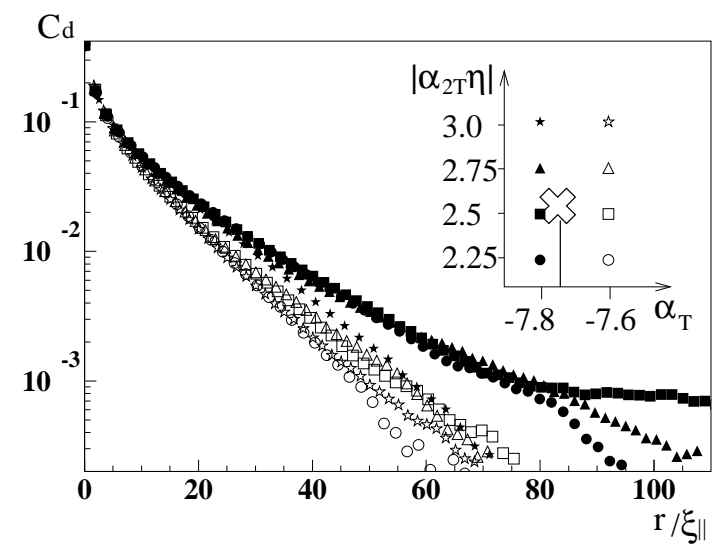

FIG. 5. Density-density correlations along the $c$-axis near the end the first order transition line and the critical end-point (see inset). Note the decreasing difference in correlations between $\alpha_{T}=-7.6$ and $\alpha_{T}=-7.8$ as the transition disappears. For $\left|\alpha_{2 T} \eta\right|=2.5$ and $\alpha_{T}=-7.8$ we see evidence for a long length scale associated with fluctuations in the average order parameter density. System sizes are $N_{a b}=72,220<N_{c}<260$ for $\alpha_{T}=-7.6$ and $N_{c}=270$ for $\alpha_{T}=-7.8$.

Density correlations diverge at the liquid-gas critical end-point on a mesoscopic scale. Analogous correlations on a larger scale appear in the numerical data between $\alpha_{T}=-7.6$ and $\alpha_{T}=-7.8$ as $\left|\alpha_{2 T} \eta\right|$ is increased to its critical end-point value. Figure 5 shows for $\left|\alpha_{2 T} \eta\right|=2.5$ and $\alpha_{T}=-7.8$ evidence of a second, much longer length scale governing the decay of the correlation function at large distances. This length scale is associated with the density fluctuations at the critical end-point and only becomes visible once it is larger than that of the vortex correlations. Due to the small amplitude of these density fluctuations very long simulation times are needed to see the correlations within the statistical noise.

\section{Anisotropic scaling and the value of $B_{l c}$}

The location of the numerical first order transition is to first approximation a line of constant $\alpha_{T}$ and thus in agreement with 3D LLL scaling. This is, although surprising for very low couplings, not unnatural in YBCO, where even for the highest fields and lowest couplings, e.g for a transition at $B=20 \mathrm{~T}, T=65 \mathrm{~K},\left|\alpha_{T} \eta\right|$ is of the order $1 / 2$ and the correlation length along the $c$-axis above the transition (see Sec. IV A 1) of the order of 10 layers. The continuum approach can thus be expected to work fairly well for YBCO.

\section{Scaling with anisotropy $\gamma$}

According to Eq. 4 the $B(T)$ dependence of a line of constant $\alpha_{T}$ ban be approximated as

$$
B \propto \frac{\left(T_{c}-T\right)^{3 / 2}}{\kappa^{2} \gamma} .
$$

Thus we expect our transition line in samples of different anisotropies to scale as $B \propto 1 / \gamma$, which is the experimentally observed scaling [12]. This form of scaling disagrees with a London-Lindemann type melting theory. The variation of the location of the low field critical endpoint with anisotropy $\gamma$ is discussed at the end of this section in the context of a general analysis of variations in $B_{l c}$.

\section{Variation with the angle of the applied magnetic field}

We cannot in our simulation change the orientation of the applied field. We can, however, using the known properties of the numerical transition discuss the expected behavior upon such a change in field orientation. We believe that the first order transition is predominantly of a decoupling nature. However, it is important to keep in mind that the coupling between layers in our simulation is not magnetic coupling of vortex segments, but Josephson coupling of the order parameter. This type of coupling is independent of the orientation of the flux lines with respect to the $c$-axis. Under angle rotation between $\theta=0(B \| c)$ and $\theta=\pi / 2(B \perp c)$, the transition line should scale according to anisotropic continuum scaling [14] with $\theta$ as

$$
B(\theta, T)=\gamma_{\theta} B(0, T),
$$

where $\gamma_{\theta}$ is given by $\gamma_{\theta}=\left(\cos ^{2} \theta+\sin ^{2} \theta / \gamma^{2}\right)^{-1 / 2}$. This form of scaling has been observed for the first order transition line in YBCO [13].

Our argument linking the nature of the transition to the location of the end-point applies for any orientation of the magnetic field. The location of the end-point is for all orientations given by $\xi_{c} \approx d$, where $\xi_{c}$ is the coherence length along the $c$-axis. For $B \| c$ this condition is equivalent to $\xi_{\|} \approx d$. Using $\xi_{\|}=\hbar /\left(2 m_{c}\left|\alpha_{H}\right|\right)^{1 / 2}$, this condition can be transformed to a simple $B(T)$ dependence which scales like the transition line itself. The end-point where both lines cross therefore equally just shifts to a higher field as $B_{l c}(\theta)=\gamma_{\theta} B_{l c}(0)$. This form of scaling has been experimentally observed for the endpoint in YBCO [13]. 


\section{Variations in $B_{l c}$}

The location of the numerical critical end-point agrees well with the experimental data of Fig. 22. However, as already mentioned in Sec. IA, the experimental value of $B_{l c}$ varies widely between different samples. Approximating $\kappa$ and $T$ as constant at the critical end-point, we obtain from Eq. 5 the scaling relation $B_{l c} \propto 1 / \gamma^{4}$. The $\kappa$ dependence is $B_{l c} \propto 1 / \kappa^{2}$. These two scaling relations show that $B_{l c}$ depends very sensitively on material parameters.

We can use the fit in Fig. 2 with $\gamma=7.5$ and $B_{l c}=0.7 \mathrm{~T}$ as reference point to compare the location of the critical end-point in our simulation to experimental values of $B_{l c}$. For samples with measured anisotropies of $\gamma=7.8$ (Ref. [13]), $\gamma=7.0,5.9,5.3$ (Ref. [15]) specific heat peaks have been measured down to $B_{l c}=0.7 \mathrm{~T}$ (Ref. 13 ), $B_{l c}=3 \mathrm{~T}$, $4.5 \mathrm{~T}, 6 \mathrm{~T}$ (Ref. [15]). The rapid increase of $B_{l c}$ with decreasing anisotropy is in qualitative agreement with a scaling law $B_{l c} \propto 1 / \gamma^{4}$.

Quantitative analysis however yields only poor agreement. The predictions using the above reference point and scaling law deviate from the experimental value by $-15 \%$ (Ref. [13]) and $-70 \%,-60 \%,-50 \%$ (Ref. [15]). There are many possible reasons other than variations in $\kappa$ for this quantitative disagreement. Firstly, the finite width of the transition due to sample inhomogeneities may lead to a spreading out of the specific heat peak, which can make it undetectable for the lowest fields above $B_{l c}$ and lead to overestimates of $B_{l c}$. Secondly any aspects of the physical coupling which are not represented in our model could lead to corrections in the effective $\left|\alpha_{2 T} \eta\right|$ and should thus be included for an accurate description.

A third, very important point is that near $T_{c}$ critical fluctuation effects arising from the zero field transition are not negligible and especially affect the divergence of $\xi_{\mu}$. Such effects extend to fields of the order of $G i \times H_{c 2}(0)$ [38, in YBCO $\sim 1 \mathrm{~T}$. Up to these fields, the LLL approximation is invalid because higher Landau levels are needed to allow for critical fluctuations. Thus the endpoint lies in a region where the LLL approximation is inadequate, and we cannot expect our estimates of the position of the critical end-point to be quantitatively accurate. However, we can expect that such fluctuations well make $B_{l c}$ smaller than estimated from the LLL approximation. Critical fluctuations cause $\xi_{\|}$to diverge faster than in the LLL approximation, so it reaches a value $O(d)$ at temperatures closer to $T_{c}$ than in the LLL approximation. This in turn will reduce the value of $B_{l c}$.

In addition, despite extensive finite size effect analysis (see appendix 9) we can never fully exclude the possibility that the location of the end-point would shift to lower fields if we used much larger systems. The existence of an end-point is fairly securely established by our physical argument. We have also made sure that the the ratio of correlation lengths to the linear system dimensions, $l_{a b} / L_{a b}$ and $l_{c} / L_{c}$, decrease rather than increase as we pass the critical end-point with increasing $\left|\alpha_{2 T} \eta\right|$. However, the general tendency of small system sizes to decrease discontinuities at the transition could have lead to an overall underestimate of the jumps due to finite size effects and thus to an overestimate of $B_{l c}$.

The presence of sample disorder increases the value of $B_{l c}$, an effect which we report for random disorder in our simulation in Sec. ฤ. Real YBCO samples exhibit in general not random disorder but clusters of oxygen vacancies and large scale sample inhomogeneities. Oxygen clusters pin at low fields a considerable fraction of the vortex matter, so that the field can be divided as $B=B_{\text {pinned }}+B_{\text {free }}$. The fraction of pinned vortices will be increased by the presence of sample inhomogeneities which provide regions at an effectively lower $\alpha_{T}$ that exhibit stronger pinning. Such a decrease in the effective field of the free vortices $\left(B_{\text {free }}<B\right)$ could considerably increase the observed $B_{l c}$.

Considering that the magnetic field at the end-point depends so sensitively on the model parameters, critical fluctuations and disorder, the good quantitative agreement with the data of Ref. [10,13] seems more than can be reasonably expected from our model, and maybe is indeed a product of chance in which $B_{l c}$ is in our simulation increased by inaccuracies of our model and/or finite size effects by the same amount that it is increased by disorder effects in the samples in Ref. 110,13.

\section{E. Beyond the critical end-point}

It is often supposed that the first order transition in YBCO changes to second order below the end-point, where no latent heat is visible but a "step" in the heat capacity $C$ remains 13,12 . We believe however that this "step" can be identified with the onset of a small rounded peak in the superconducting specific heat $C_{s}=C-C_{n}$, ( $n$ for normal state), which is known to arise from thermal fluctuations 39]. In this section we shall examine experimental specific heat data and numerical data in the light of this possibility.

The specific heat peak due to thermal fluctuations has been observed for example in niobium by Farrant and Gough 40], where observations are in good agreement with theoretical predictions [39]. We find that the location and the height of the peak as well as the length of the rise (or width of the "step") in $C$ from the low temperature value $C_{s, m f}$ ( $m f$ for mean-field) to its maximum agree well for the niobium and YBCO measurements taken from Ref. 40] and [13. We shall now explain in detail how the data from both references can be compared and give respectively values for the location, width and height of the specific heat peak or "step". 
For a clearer picture of how the experimentally measured specific heat splits into normal and superconducting contribution as well as how it compares to the mean-field contribution, a schematic plot is given in Fig. 6 .

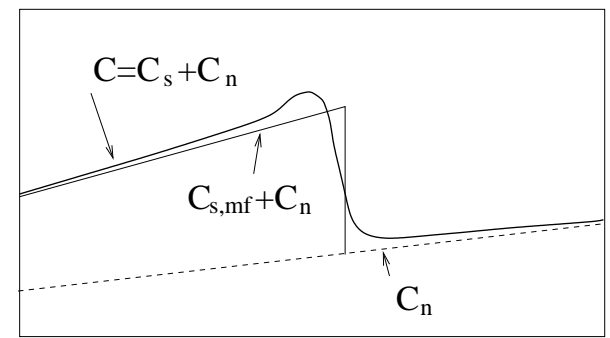

FIG. 6. Schematic view of a typical experimental specific heat curve without first order peak (thick line) with normal state contribution (dashed line) and mean-field value (thin line).

Farrant and Gough give in Ref. [40] the superconducting specific heat data already in terms of LLL scaling parameters. The plotted quantity is $C_{s} / C_{s, m f}$. The data in Fig. 3 from Ref. 13 which shows the specific heat in YBCO is given as $C$ minus $C(B=0)$. The latter near the "step" is approximately equal to the low temperature value $C_{s, m f}+C_{n}$, because for $B \rightarrow 0, \alpha_{T} \rightarrow-\infty$. This approximate equality is also visible in Fig. 1 of the same Ref. [13]. The plotted quantity, $C-C(B=0)$, is therefore approximately equal to $C_{s}-C_{s, m f}$.

The peak in $C_{s}$ in niobium obeys LLL scaling and is found at $\alpha_{T} \approx-7$. The specific heat maximum in YBCO occurs at temperatures just above the extrapolation of the first order transition line, which is located at $\alpha_{T} \approx-7.8$. For the example curves for $B=0.25 \mathrm{~T}$ and $B=0.5$ in Fig. 3 from Ref. [13], the center of the broad specific heat peak is at $T \approx 91.4 \mathrm{~K}$ and $T \approx 90.7 \mathrm{~K}$ respectively, which both translate with the same previously used YBCO material constants to $\alpha_{T}=-7.2$. This is in very good agreement with niobium.

The width of the specific heat rise in niobium $\Delta \alpha_{T} \approx 2$. For $B=0.25 \mathrm{~T}$, no sharp step feature is visible in the YBCO data. The specific heat rise from the low temperature value to the maximum takes place in the temperature region $91-91.4 \mathrm{~K}$, which corresponds to $\Delta \alpha_{T}=2.8$, broader than in niobium. For $B=0.5 \mathrm{~T}$ one might suspect a step-like feature located $90-90.5 \mathrm{~K}$. This width corresponds to $\Delta \alpha_{T}=2.3$, a value in agreement with the niobium data.

For niobium $C_{s}$ is at its maximum $5 \%$ larger than $C_{s, m f}$. In YBCO we have to divide the plotted data by $C_{s, m f}$ to compare with this value. $C_{s, m f}$ is roughly given by the step in $C$ at the zero field transition, which we take from Figure 1 of Ref. 13 as $C_{s, m f} \approx$ $60 \mathrm{~mJ} / \mathrm{mole} \mathrm{K}^{2}$. For all fields the specific heat "step" in YBCO is of the order of $1.5 \mathrm{~mJ} / \mathrm{mole}^{2}$, which gives $\left(C_{s}-C_{s, m f}\right) / C_{s, m f} \approx 2.5 \%$, which we consider as reasonable agreement with in niobium. Exact agreement cannot be expected for the following reasons. The value of $C_{s, m f}$ we used for YBCO is a rather crude approximation. For niobium $C_{s, m f}$ is also somewhat uncertain, because it depends on the choice of $C_{n}$ (see Fig. 6), which is not directly measurable but extrapolated from fits to higher temperature data. Also the data in YBCO at fields below $B_{l c}$ are in a region where the LLL approximation is inadequate.

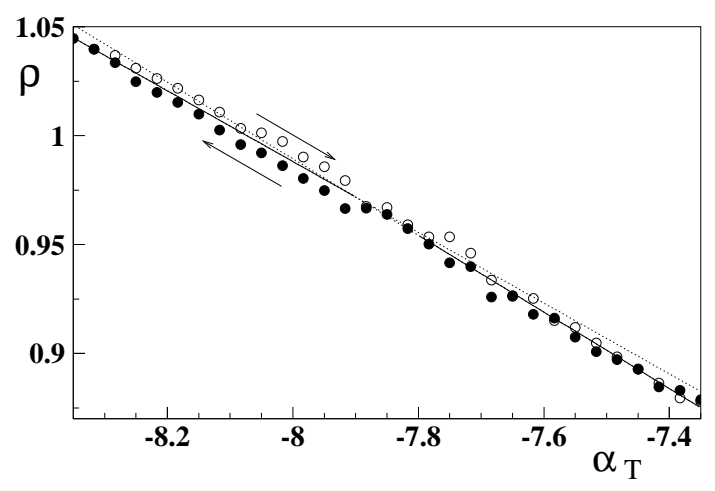

FIG. 7. Plots of the order parameter density $\rho$ upon heating and cooling for $\left|\alpha_{2 T} \eta\right|=3$. Solid lines are linear fits for the regions above and below the extrapolation of the first order transition line, extrapolated as dotted lines. $N_{a b}=72$, $N_{c}=80$.

If the step feature is mainly due to the increase of LLL fluctuations, it should be observable in our simulation. A "step" in the superconducting specific heat corresponds to a change in the slope of the magnetization, in simulation terms a change of $\partial \rho / \partial \alpha_{T}$. Figure 7 shows $\rho$ upon cooling and heating for $\left|\alpha_{2 T} \eta\right|=3$, well beyond the critical end-point. The data for $\alpha_{T}<-7.9$, i.e. below the region where the first order transition takes place in more layered samples, is very strongly affected by hysteresis. The sweep rate being of the same order as usual, this is a sign of very long fluctuation time scales. We shall come back to this point in Sec. IV. Linear fits to the data for both cooling and heating below and above the extrapolated transition line, $\alpha_{T}<-7.9$ and $\alpha_{T}>-7.8$, give a change in slope of $8 \%$, larger than in the experimental data from both niobium and YBCO.

In summary we have seen that the specific heat "step" in YBCO at different fields has approximately the same amplitude as well as width and position when expressed in terms of $\alpha_{T}$, i.e. the "step" feature obeys LLL scaling (LLL scaling has for the steps associated with the first order transition previously been established in Ref. [37). The corresponding data from our numerical simulation are not equilibrated and therefore not very accurate, but consistent with a similar "step" feature. The semi-quantitative agreement between YBCO and niobium strongly suggests that we are dealing with the same phenomenon and therefore that there really is no sharp 
specific heat step in YBCO beyond the critical end-point.

\section{ORDER PARAMETER CORRELATIONS}

The existence of the critical end-point implies that no symmetries are broken at the transition, which means it cannot be a liquid-crystal transition. Investigation of the nature of order parameter correlations described in this section confirms that the state below the transition is a vortex liquid. However, neutron diffraction patterns corresponding to a triangular lattice and an electrical resistance close to zero below the first order transition line suggest very long-range vortex correlations. We shall argue that the extremely fast growth of correlation length scales and relaxation time scales below the first order transition, which have been theoretically predicted in the low temperature regime [41] as well as observed in our simulation, can account for these effects.

\section{A. Static order parameter correlations}

We have measured various different kinds of equilibrium order parameter correlation functions, including the structure factor as previously defined in Ref. 32] and given by the normalized density-density correlator in Eq. 11 (below) for $\Delta r=0$ along the $c$-axis. The peaks in the structure factor, which occur at the reciprocal lattice vectors of the triangular lattice, reflect the crystalline correlations within each layer. To examine order parameter correlations along the $c$-axis, we have measured three different correlators. Firstly, we measured the densitydensity correlations along a line parallel to the magnetic field, $C_{d}(\Delta r \| c)$, the same correlator that gave in Sec. III evidence of a diverging length scale at the critical end-point. Secondly, we measured the decay of the $a b$ Fourier transformed density-density correlations, which are for $\Delta r \| c=0$ the structure factor of the system, depending on $\Delta r \| c$. Just as for the structure factor [32] we normalize by the high temperature limit, so that the relevant quantity is

$$
C_{d}(k\|a b, \Delta r\| c) / \lim _{\alpha_{2 T} \rightarrow \infty} C_{d}(k \| a b, 0),
$$

with

$$
\begin{aligned}
& C_{d}(\mathbf{k}\|a b, \Delta \mathbf{r}\| c)= \\
& \frac{1}{\left\langle|\psi|^{2}\right\rangle^{2}}\left(\left\langle|\psi|^{2}(\mathbf{k}, \mathbf{r})|\psi|^{2}(-\mathbf{k}, \mathbf{r}+\Delta \mathbf{r})\right\rangle-\right. \\
& \left\langle|\psi|^{2}(\mathbf{k}, \mathbf{r})\right\rangle\left\langle|\psi|^{2}(-\mathbf{k}, \mathbf{r}+\Delta \mathbf{r}\rangle\right) .
\end{aligned}
$$

The definition of this correlator in its generalized, time dependent form in terms of the LLL coefficients and the high temperature limit are given in Sec. B2, Eq. B2 and B3. We find that this correlator decays most slowly with
$\Delta r \| c$ for $k_{a b} \approx G$, the first reciprocal lattice vector. In addition to $C_{d}$ we also measured the phase correlator $C_{p}$ defined as

$$
C_{p}(\boldsymbol{\Delta} \mathbf{r} \| c)=\alpha_{T} \frac{\beta\left\langle\psi^{*}(\mathbf{r}) \psi(\mathbf{r}+\boldsymbol{\Delta} \mathbf{r})\right\rangle}{2 \pi \alpha_{H}},
$$

which is expressed in terms of the LLL coefficients in Sec. B2. The prefactor is chosen such that that $C_{p}(0)$ is the order parameter density $\rho$. In Fig. 8 we show examples of static density and phase correlations above and below the transition for $\left|\alpha_{2 T} \eta\right|=1$, which corresponds to a transition temperature of $83 \mathrm{~K}$ in YBCO.
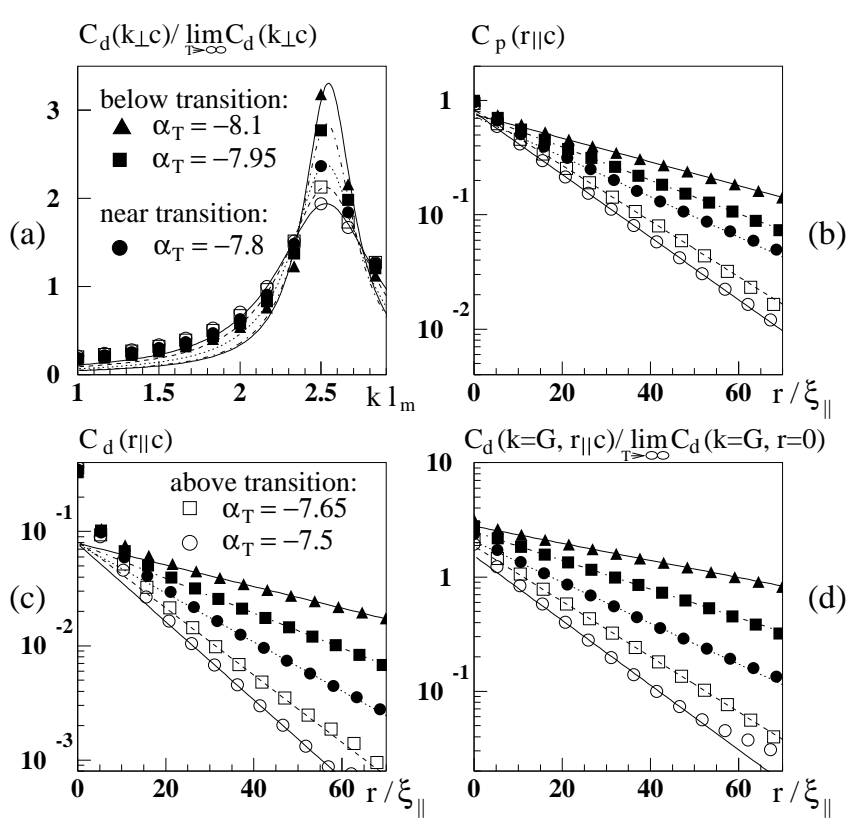

FIG. 8. Static order parameter correlations above and below the first order phase transition at $\left|\alpha_{2 T} \eta\right|=1$. (a) Structure factor (with Lorentzian fits), (b) phase correlations along the $c$-axis. Plots (c) and (d) show density-density correlations along the $c$-axis, where the correlator in (c) is in real space for points with the same coordinates in the $a b$-plane, and the correlator in (d) is in Fourier space near the first reciprocal lattice vector of the triangular lattice, $G$. Note that in plots (b),(c),(d) the $y$-axes are such that parallel linear fits correspond to the same length scale. The growth of length scales is slowest for the phase correlations in (b) and fastest in (d). The system size is $N_{a b}=72, N_{c}=270$.

Figure $8(a)$ shows examples of the structure factor with Lorentzian fits. The inverse width at half maximum, $\delta^{-1}$, of the peak near the first reciprocal lattice vector is proportional to the crystalline correlation length $l_{a b}$ within one layer [32], $\delta^{-1}=l_{a b} / 2 l_{m}$. No qualitative change in behavior is visible across the transition, just sharpening of the peak, which corresponds to an increase of $l_{a b}$. For the above fits $l_{a b} / l_{m}=2.66,3.14,3.88$, $4.94,5.46$. This is shorter than the radius of the sphere 
$R / l_{m}=\sqrt{N / 2}=6$ and shorter than the average distance between the 12 disclinations imposed by the spherical geometry, $\sqrt{4 \pi R^{2} / 12} \approx R$. This means that finite size effects on this data are not expected to be too large.

Figures 8(b)-(d) show examples of measurements of the three different correlators along the c-axis. Figure 8(b) shows the phase correlator, Fig. 8(c) the real space density-density correlator and Fig. 8(d) the $a b$-Fourier transformed density-density correlator near the first reciprocal lattice vector $G$. In all cases we see an exponential decay of the correlation function with a finite length scale $l_{c}$ for density correlations below as well as above the transition. Only for a liquid phase would these correlation functions all have an exponential decay.

All three correlators decay over half the system size $n<N_{c} / 2$, and then rise to $C\left(N_{c} d\right)=C(0)$ due to the periodic boundary conditions. To extract decay length scales we fit to the first portion, approximately $\Delta r<\left(N_{c} d / 6\right)$, of the decay, as indicated by linear fits. This region of decay is least affected by finite size effects, statistical noise and errors due to incomplete equilibration (see Sec. Q). The length scales extracted from the linear fits are of the same order for all three correlators. Just above the transition the different $l_{c}$ are almost equal. Below the transition the Fourier transformed densitydensity correlations clearly have the largest length scale with $l_{c, d} / \xi_{\|}=58$ ( $d$ for density) at $\alpha_{T}=-8.1$ and the phase correlator the smallest with $l_{c, p} / \xi_{\|}=42$ ( $p$ for phase) at the same $\alpha_{T}$.

At temperatures well above the transition we find the opposite behavior. The density-density correlation length is at temperatures $\alpha_{T} \approx-4$ little more than half of the phase correlation length, which agrees with the simple high temperature expectation $\left\langle\psi(r)^{*} \psi\left(r^{\prime}\right)\right\rangle^{2} \sim$ $\left\langle|\psi(r)|^{2}\left|\psi\left(r^{\prime}\right)\right|^{2}\right\rangle$ and therefore for exponential decay $l_{c, p} \approx 2 l_{c, d}$. However, because we find that below the transition $l_{c, d}$ is the longest and therefore dominant length scale, we shall in the following refer to the decay length scale of the correlator $C_{d}\left(k_{a b}=G\right)$ as $l_{c}$.

\section{Temperature dependence of correlation lengths}

In Fig. 9 we plot on the left the $\alpha_{T}$ dependence of the inverse width at half maximum of the first peak in the structure factor, $\delta^{-1}$, which is proportional to the length scale of in-plane crystalline order, $l_{a b} / l_{m}=2 \delta^{-1}$, and on the right the length scale $l_{c}$ as obtained from linear fits to the decay of density-density correlations. The length scales have a discontinuity at the transition, which is found to grow with distance from the end-point, as one would naturally expect. This discontinuity is clearly visible for $\left|\alpha_{2 T} \eta\right|=0.05$. At large couplings, finite size effects spread out the discontinuity. The data for $\left|\alpha_{2 T} \eta\right|=1$ shows a rapid growth of the length scales at and below the transition.
If the phase coherence of the Abrikosov state is examined in the presence of thermal fluctuations, one finds that in and below three dimensions thermal fluctuations destroy phase coherence at any finite temperature [42]. Under the assumption that there is only one relevant length scale describing phase order and crystalline order in the system, perturbative studies for the continuum low temperature regime predict an exponential growth of length scales with $\left|\alpha_{T}\right|^{3 / 2}$ as $l_{c} \propto \exp \left(A\left|\alpha_{T}\right|^{3 / 2}\right)$ and $l_{a b} \propto \exp \left(0.5 A\left|\alpha_{T}\right|^{3 / 2}\right)$ 41. The authors of Ref. 41] estimate that $A$ may be given by its upper limit value $A=0.53$. The slope of such growth behavior with $A=0.53$ is given by the solid lines in Fig. 9 . The growth rate in our simulation data is at the lowest temperatures of the same order as the theoretically predicted upper limit. However, the analytical result is from an expansion around zero temperature in the continuum limit. This regime is for numerical reasons described in Sec. I not accessible to our simulation. Therefore perfect agreement of our simulation results with this form cannot be expected.
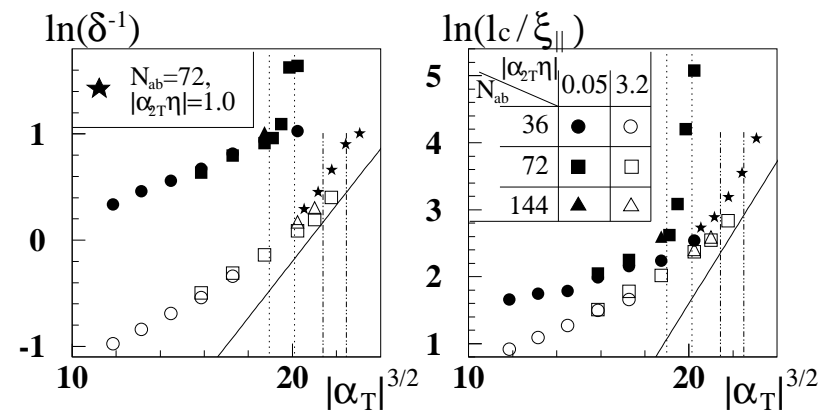

FIG. 9. Logarithm of correlation lengths plotted against $\left|\alpha_{T}\right|^{3 / 2}$. Solid lines represent the growth rate for the low temperature regime as predicted from perturbative expansions around zero temperature [41]. Dotted and dot-dashed lines mark the width of the first order phase transition from the magnetization discontinuity for $\left|\alpha_{2 T} \eta\right|=0.05$ and $\left|\alpha_{2 T} \eta\right|=1$ respectively. Note the fast growth of length scales for $\left|\alpha_{2 T} \eta\right|=1$ below the phase transition. Note also that the data below the transition for $\left|\alpha_{2 T} \eta\right|=0.05$ is strongly affected by finite size effects $\left(l_{a b} \approx 10 l_{m}>R=6 l_{m}\right)$.

The data in Fig. 9 for $\left|\alpha_{2 T} \eta\right|=3.2$, which is beyond the critical end-point and has got no phase transition, suggests that a faster growth of length scales sets in approximately where the phase transition is located in more layered samples. The reason why we have obtained no more data at lower temperatures to confirm this tendency is just because of the fact that length and time scales (see Sec. IV B) grow so fast that we found it impossible to equilibrate a sufficiently large system at even lower temperatures. (Remember that the same system size in units of $\xi_{\|}$corresponds to more layers for larger $\left|\alpha_{2 T} \eta\right|$.) However, the onset of fast growth of length scales can 
be expected from the fact that in the low temperature regime, where length scales are so long that the continuum approximation works well even for strongly layered materials, the data for different $\left|\alpha_{2 T} \eta\right|$ must collapse onto a single curve and depend only on $\alpha_{T}$. At the transition, length scales in the more continuous samples are in the appropriate scaling units distinctly shorter than for low $\left|\alpha_{2 T} \eta\right|$. For the length scales in these systems to approach the lengths scales of the more layered systems, rather fast growth is necessary not far below the transition.

\section{The appearance of Bragg peaks}

Just below the phase transition the correlation length scales in the simulation are for the range of coupling parameters that correspond to YBCO not comparable to the much larger length scales needed to give a signal in neutron diffraction experiments. Although the structure factor in a liquid is rotationally symmetric, coupling with the underlying lattice or preferred orientations given by twin planes may for long length scales lead to the appearance of Bragg-like peaks 443].

Our simulation suggests that the vortex liquid is not far below the first order transition correlated and effectively crystalline over length scales comparable to the system size or a "Larkin"-like length scale (dependent on the amount of disorder present). We can extrapolate the growth in length scales in Fig. 9 below the transition assuming the exponential growth rate estimated in Ref. 411 and indicated by the solid lines. For a decrease of $\Delta \alpha_{T} \approx 1.2$ for example, which corresponds in YBCO to cooling by only $1 \mathrm{~K}$ or $(1 / 4) \mathrm{K}$ below the transition at $5 \mathrm{~T}$ or $0.7 \mathrm{~T}$ respectively, we obtain an increase in $l_{a b}$ by a factor of 4 and an increase in $l_{c}$ by a factor of 16 . At $\alpha_{T} \approx-10.5$ the crystalline correlation length $l_{a b}$ has according to the same estimate reached 30 lattice spacings and $l_{c}$ the order of $10,000 \xi_{\|}$, which is for magnetic fields of $B \approx 5 \mathrm{~T}$ or $0.7 \mathrm{~T}$ of the order of 5,000 and 10,000 layers respectively. Correlation lengths of this order can be expected to lead in real samples to observable neutron diffraction peaks.

Figure 10 shows scaling plots of different experimental phase diagrams in different YBCO samples. We expect according to LLL scaling that the plots of $\gamma B(T)$ from samples with a different mass anisotropy but otherwise identical material parameters collapse (see Sec. IIIB]). Roulin et al. [12] report that the first order transition lines in different clean samples, represented by a solid line in Fig. 10, as well as the the line of steepest slope at the specific heat step in disordered samples [15], represented by a dashed line in Fig. 10, collapse in this way. The solid and the dashed line correspond to the specific heat peaks in three different samples with different anisotropies each. The Schilling data (scaled using $\gamma=7.8$ [13]) also agrees reasonably well with this scaling form.

According to the vortex lattice melting picture, the points which mark the onset of neutron diffraction peaks in Fig. 10 (scaled using $\gamma=4.3$ [20]), should coincide with the first order transition line. Comparison however shows that $\gamma B$ is roughly a factor 2 below the first order transition lines. (In a scaling plot of $\gamma^{2} B$, which should yield a collapse assuming London-Lindemann-type melting, the discrepancy between the Aegerter and Schilling data would be even larger, roughly a factor 4.) Very substantial differences in other material parameters would be necessary to make up for such a large deviation. In our picture the relative position of the two features is natural, because the onset of neutron diffraction peaks is attributed to a crossover below the first order transition, when the neutron diffraction experiment becomes sensitive to the exponentially fast diverging length scales.

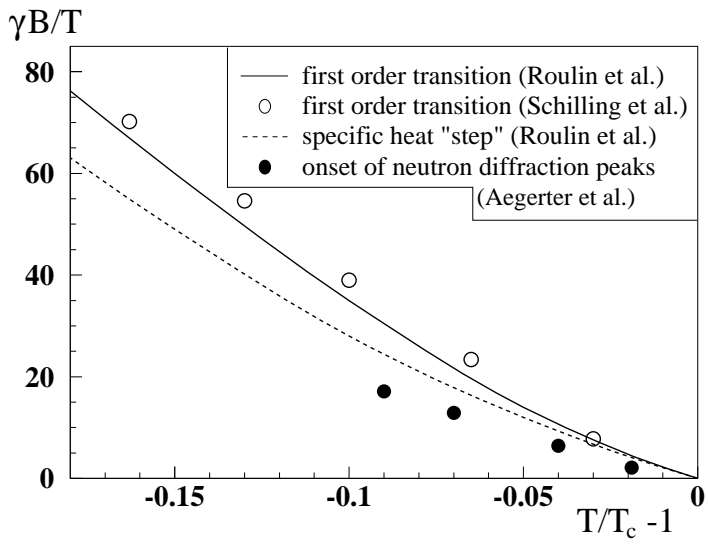

FIG. 10. Comparison of phase diagrams obtained on different samples from thermodynamic and neutron diffraction measurements. All data is for $B \| c$. Empty circles and the solid line (extrapolated to $T_{c}$ beyond $B_{l c}$ ) correspond to specific heat peaks. The dashed line represents the point of steepest slope of the specific heat "step" attributed to second order melting in samples which do not show a specific heat peak. The data for the solid line, empty circles, the dashed line and filled circles is taken from Ref. [12,13, 15,20] respectively.

However, one might say that comparing first order "melting" and onset of neutron diffraction is not a comparison of like with like, because the sample in the neutron diffraction experiment is far too dirty to exhibit a first order phase transition. The authors of Ref. 20] estimate that their sample is comparable to samples from Ref. [15] and attribute the onset of neutron diffraction peaks to second order freezing to a vortex glass. In this case they should coincide with the line marking position of the steepest slope of the specific heat steps in such samples, which has been interpreted as a second order vortex glass transition 15]. However, the plots in Fig. 10 show that this is not the case. 
Our scaling approach is based on the approximation $\alpha_{T} \sim 1 /(\gamma B)^{2 / 3}$. Assuming that the first order transition line found by Roulin et al. [12] occurs as in our simulation at $\alpha_{T} \approx-7.5$, this means that the dashed line and the onset of neutron diffraction in Fig. 10, for which $\gamma B$ is reduced by a factor of about $3 / 4$ and $1 / 2$ respectively, correspond to $\alpha_{T} \approx-9$, and $\alpha_{T} \approx-10.5$ respectively. The $\alpha_{T}$ value for the onset of neutron diffraction peaks is in excellent agreement with our estimates as to where $l_{c} \approx 10,000 \xi_{\|}$and $l_{a b} \approx 30$ lattice spacings in the clean limit.

\section{B. Relaxation times}

The analysis of relaxation times is numerically difficult. It turns out that the dominant relaxation times are very large near the first order phase transition. Accordingly thermal averaging is slow and measurements of the time decay of the density-density correlator have large statistical errors. However, although our data on relaxation times is of rather poor quality, it is still of interest for comparison with the analysis of the vortex dynamics in the 2D system as well as for comparison with nonequilibrium measurements in YBCO.

As in our previous analysis of the $2 \mathrm{D}$ system [35], we measure the relaxation time scales in the layered system from the decay of the density-density correlator $C_{d}$ from Eq. 10 in its Fourier transformed time-dependent form, $C_{d}\left(k_{a b}, k_{c}, t\right)$. We observe for high temperatures linear exponential decay of this correlator to zero for all $k$, where the $k_{a b}$ dependence of the decay time scales reflects, like in 2D [35], the hexagonal order in the system. The time scales decrease monotonically with $k_{c}$ for all $k_{a b}$. For low temperatures, the time scales over which we measure the decay are often much smaller than the longest decay time scales themselves, so that decay of the correlator is only observable over a fraction of its initial value. However, we can still, knowing that the vortex matter is liquid, extract time scales by fitting the data assuming linear exponential decay. The longest time scales in the system are in all cases given by the decay of the $3 \mathrm{D}$ Fourier component of $C_{d}$ at the first reciprocal lattice vector in the $a b$-plane and $k=0$ along the $c$-axis.

Figure 11 shows a plot of the dominant time scales depending on $\left|\alpha_{T}\right|^{3 / 2}$ for different $\left|\alpha_{2 T} \eta\right|$. The plots suggest very fast growth behavior, roughly

$$
\tau \sim \exp \left(c_{1} \exp \left(c_{2}\left|\alpha_{T}\right|^{3 / 2}\right)\right)
$$

where $c_{1}$ and $c_{2}$ are appropriate constants. We expect an exponential growth of the dominant time scales with the range of correlations, $\tau \propto \exp F l_{a b}^{\psi}$, if the dynamics is activated, as it is in the $2 \mathrm{D}$ limit 35. The data in Fig. 11 together with the low temperature relation $\ln \left(l_{c}\right) \propto 2 \ln l_{a b} \propto\left|\alpha_{T}\right|^{3 / 2}$ 41, which is approximately also valid for our numerical data (see Fig. 9), strongly suggests such activated dynamics in the layered system.

Figure 11 shows evidence that time scales increase discontinuously across the transition. If activated scaling of the type $\tau \sim \exp \left(c_{1} \exp \left(c_{2}\left|\alpha_{T}\right|^{3 / 2}\right)\right)$ holds, the discontinuity in length scales should be amplified in the time scale discontinuity. The increase in $\tau$ across the transition for $\left|\alpha_{2 T} \eta\right|=0.05$ is roughly a factor 500 . In the case $\left|\alpha_{2 T} \eta\right|=1$ the time scales increase across the transition according to our fits only by a factor 4 . Although we expect the discontinuity in time scales to decrease as $\left|\alpha_{2 T} \eta\right|$ increases and the end-point is approached, it is very likely that we strongly underestimate the time scales of exponential decay below the transition for $\left|\alpha_{2 T} \eta\right|=1$, because we fit to a very small regime of decay (see inset of Fig. 11). As in the 2D case [35] the relaxation behavior at very early times is faster than the final, linear exponential decay.

As in the case of length scales, the data for all $\left|\alpha_{2 T} \eta\right|$ should collapse at low $\alpha_{T}$, i.e. in the continuum limit. This implies very fast growth of time scales below the transition $\alpha_{T}$ for continuous systems with $\left|\alpha_{2 T} \eta\right|$ beyond the end-point. This extremely fast growth is reflected in the behavior of the system upon heating and cooling for $\left|\alpha_{2 T} \eta\right|=3$ (see Fig. 7), where strong hysteresis due to very slow decay of thermal fluctuations is observable below $\alpha_{T}=-8$.

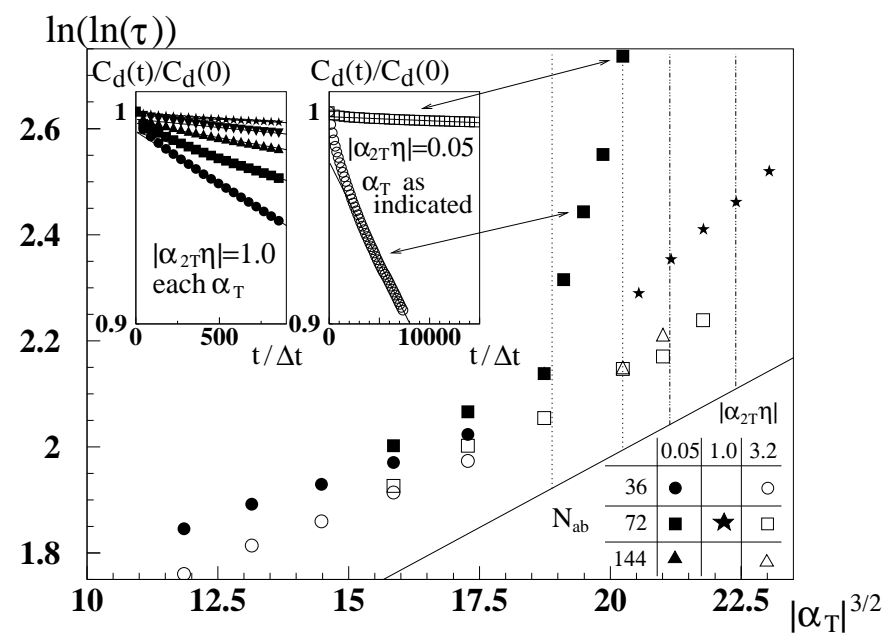

FIG. 11. Logarithm of logarithm of relaxation times plotted against $\left|\alpha_{T}\right|^{3 / 2}$. Dotted and dot-dashed lines mark the width of the first order phase transition from the magnetization discontinuity for $\left|\alpha_{2 T} \eta\right|=0.05$ and $\left|\alpha_{2 T} \eta\right|=1$ respectively. The inset shows logarithmic plots of original relaxation data with linear fits.

\section{Comparison with resistivity data}

Although the relevant time scales for dc resistance measurements in $\mathrm{YBCO}$ are non-equilibrium pinning 
time scales which are not directly comparable to the relaxation times measured in the simulation, some connections can be made. While our time scales are irrelevant for flux flow conductivity, they are relevant in a highly viscous vortex liquid regime, where the time scales of thermal fluctuations or plastic deformations of the vortex structure are much larger then the pinning time. In this case one speaks about a pinned vortex liquid [3]. In such a liquid flux flow is strongly suppressed by pinning. When there is a large discontinuity of relaxation time scales at the first order transition, it is conceivable that the flux liquid becomes suddenly pinned and discontinuities in the resistive behavior occur.

A decrease of the discontinuity in time scales as one approaches the critical end-point agrees qualitatively well with the decreasing discontinuity in the resistive data as $B_{l c}$ is approached [16]. The decrease and complete disappearance of the resistivity jump are difficult to explain with the assumption that the first order vortex lattice melting changes to a vortex glass melting transition at $B_{l c}$. A change in resistive behavior and a discontinuity in the resistivity at low currents should remain for such a transition [3].

In experiment a very steep drop of resistivity to zero with decreasing temperature is observed even for $B<$ $B_{l c}$, when there is no first order transition. This feature can in our picture be attributed to the doubly exponentially fast divergence of decay time scales with decreasing temperature once the extrapolation of the transition line is crossed. Although there is no time scale discontinuity, the system does a fast crossover from flux flow conductivity to a strongly pinned liquid regime.

\section{Irreversibility and magnetization measurements}

A puzzling feature of the magnetization measurements near the first order transition in YBCO is the occurrence of reversible-looking magnetization jumps without the corresponding latent heat 11] which must according to the Clausius-Clapeyron relation (Eq. 9) occur for an equilibrium measurement. In Ref. [11] large magnetization jumps which would correspond to entropy jumps of up to $11 k_{B} /$ vortex/layer, more than 10 times higher than the largest jumps observed in calorimetric measurements of approximately $0.8 k_{B} /$ vortex/layer [12,13], are found without any evidence of a latent heat.

If magnetization and specific heat data do not obey the Clausius-Clapeyron relation, this must be due to lack of equilibration in the system. The connection between seemingly reversible features in the magnetization data and irreversibility has in some cases been shown experimentally. For the vortex transition in BSCCO Farrell et al. [14] found that the magnitude of the magnetization jump is for standard SQUID measurement techniques strongly correlated to irreversibility, even if irreversibility only becomes obvious at temperatures below the magnetization jump. A similar effect in YBCO has been reported by Schilling et al. 445, where a change in slope of the seemingly reversible magnetization is shown to be an effect of irreversibility.

Very recent data in YBCO by Ishida et al. in the second of Ref. [46] shows magnetization jumps in a geometry $B \| a$ at $B=1.5 \mathrm{~T}$, while in this geometry the specific heat jump has in similar samples been observed to disappear between $6 \mathrm{~T}$ and $4 \mathrm{~T}$ [13], a value in good agreement with our simulation data, if anisotropic scaling is assumed to apply. In Ref. [46] the ac $(f=390 \mathrm{~Hz})$ susceptibility is also measured and found to be an almost perfect image of the dc magnetization jump. This analogous behavior of the jumps in dc and ac susceptibility, of which the latter is clearly not a thermodynamic but a dynamic phenomenon due to non-equilibrium pinning effects [46], is suggestive of irreversibility effects even in the dc case.

Although our simulation does not reproduce these effects, the relaxation time scales we measure give a clear indication why such irreversibility related effects can occur just below the extrapolation of the first order transition line, i.e. at $\alpha_{T} \leq-8$. Let us assume that the growth in relaxation time scales is of the type $\tau \sim$ $\exp \left(c_{1} \exp \left(c_{2}\left|\alpha_{T}\right|^{3 / 2}\right)\right)$ and further take an estimate of the slope of $\ln \ln \tau$ below $\alpha_{T}=-8$ from the low temperature data in Fig. 11 for coupling $\left|\alpha_{2 T} \eta\right|=3.2$ (beyond the endpoint). Using the estimate $\partial(\ln \ln \tau) / \partial\left(\left|\alpha_{T}\right|^{3 / 2}\right) \approx 0.09$ let us consider a decrease in temperature from $\alpha_{T}=-8$ to $\alpha_{T}=-10$. This corresponds to a temperature decrease of less than $0.3 \mathrm{~K}$ below the the extrapolation of the first order line at a field of $B \| c=0.25 \mathrm{~T}$ or, according to anisotropic scaling with $\gamma=7$, to a field $B \| a$ of $1.75 \mathrm{~T}$. The considered temperature interval is thus comparable to the width of the magnetization discontinuity in the data from Ref. 46]. For this decrease in $\alpha_{T}$ we can extrapolate an increase in time scales from $\ln \ln \tau \approx 2.3$ to $\ln \ln \tau \approx 3.1$, which is by a factor $10^{5}$. For an increase of time scales by a further factor $10^{5}$ a further decrease of temperature by only $0.1 K$ is needed. This implies that cooling by only fractions of a Kelvin below $\alpha_{T}=-8$ for fields $B<B_{l c}$ can cause the system to fall out of equilibrium.

If one believes that falling out of equilibrium can lead to spurious magnetization jumps, then one may ask why a magnetization discontinuity is not always present, but in many cases disappears at low fields. For the data of Schilling et al. 10,13. magnetization and specific heat data are found to be in good agreement with the Clausius-Clapeyron relation. A possible answer may be that the size of the magnetization discontinuity caused by non-equilibrium effects depends sensitively on the presence of sample disorder. Such effects may make it negligible against the thermodynamic magnetization discontinuity at the first order transition in some samples like the ones used by Schilling et al. 10,13, but not in others. 


\section{EFFECTS OF DISORDER}

We have investigated the effects of quenched random disorder on the layered system. We have limited our study to point disorder, i.e. the disorder realizations were always uncorrelated in different layers. We shall describe the effects of disorder on the first order transition as well as on correlations.

\section{A. Effects on the first order transition}
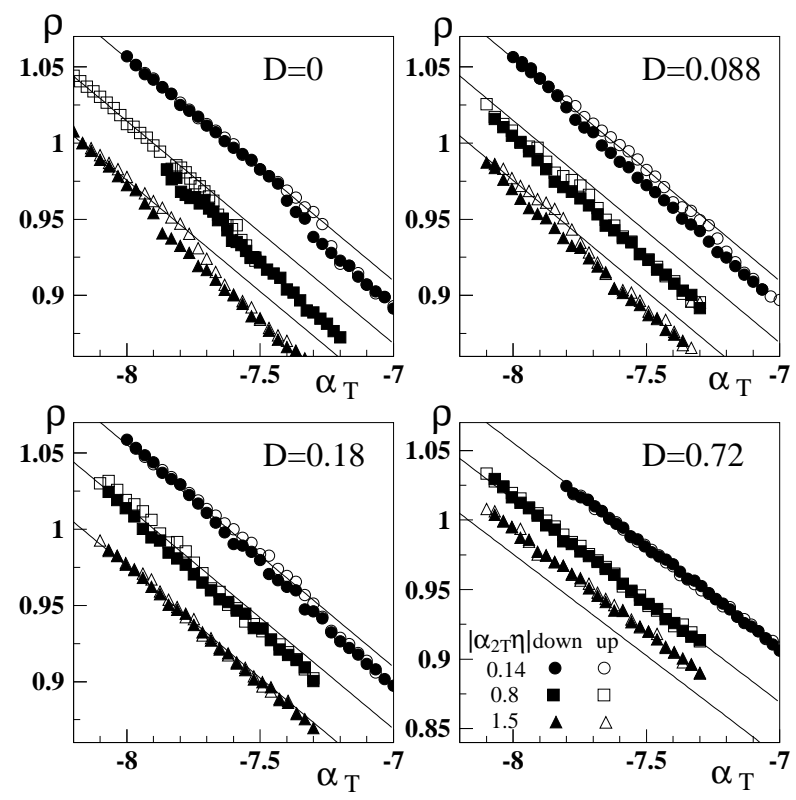

FIG. 12. Plots of the order parameter density $\rho$ upon heating and cooling for different values of disorder. Solid lines (identical for all $D$ ) are for easier comparison. The hysteresis decreases with increasing disorder, slightly faster for high $\left|\alpha_{2 T} \eta\right|$. For $\left|\alpha_{2 T} \eta\right|=0.14 N_{c}=20 \ldots 16$ (increasing with $D$ ), for $\left|\alpha_{2 T} \eta\right|=0.8 N_{c}=60 \ldots 40$ (decreasing with $D$ ), and for $\left|\alpha_{2 T} \eta\right|=1.5 N_{c}=60 \ldots 50$ (decreasing with $D$ ). For $\left|\alpha_{2 T} \eta\right|=1.5 \rho$ is offset by -0.025 .

Figure 12 shows plots of the order parameter density $\rho$ upon heating and cooling for different values of disorder strength and coupling values varying from low coupling $\left(\left|\alpha_{2 T} \eta\right|=0.14\right)$ to values near the end-point $\left(\left|\alpha_{2 T} \eta\right|=\right.$ 1.5). We observe that the discontinuity persists in weak disorder, but decreases in amplitude and increases in width upon increasing disorder until it disappears entirely. The statistical noise makes it difficult to determine the exact value of disorder for which the discontinuity disappears. However, it looks as if for $D=0.18$ the discontinuity near the end-point $\left(\left|\alpha_{2 T} \eta\right|=1.5\right)$ disappears, while for lower coupling values it is still present. This behavior would suggest that in a weakly disordered sample $B_{l c}$ can be higher than in the clean case; for the case $D=0.18$ we would expect $B_{l c}$ between $\left|\alpha_{2 T} \eta\right|=1.5$ and 0.8 , i.e. roughly between 2 and 5 Tesla in YBCO.

Our simulation does not capture the existence of an upper critical field $B_{u c}$. This can be interpreted as a sign that the appearance of $B_{u c}$ is strongly linked to the presence of disorder clusters of a typical size, which are not present in a simulation with random Gaussian disorder. There is some experimental evidence for the link of $B_{u c}$ to the clustering of oxygen vacancies. On the one hand it has been shown that the location of $B_{u c}$ in YBCO is strongly correlated with the field strength for which the fishtail effect occurs at lower temperatures [17]. On the other hand, the existence of the fishtail effect itself has been shown to be due to the presence of clusters of oxygen vacancies. A recent experiment by Erb et al. 47] demonstrates that for constant oxygen vacancy density the fishtail effect can be suppressed by the destruction of vacancy clusters.

This picture can be supported by the following qualitative argument. Let us assume the existence of a disorder length scale $l_{d}$ and consider the relation of $l_{d}$ to $l_{m}$, the length scale which characterises the average vortex spacing and in the LLL also the order parameter coherence length. If $l_{m}>l_{d}$, the system is only sensitive to a spatial disorder average, and the disorder can be considered as effectively weak. When $l_{m}<l_{d}$, the system is sensitive to the length scale of disorder, and disorder effects are not weakened. We can define a field $B_{u c} \sim \Phi_{0} / l_{d}^{2}$. Then the disorder is effectively weak for $B<B_{u c}$, which is equivalent to the relation $l_{m}>l_{d}$. In our simulation, disorder is equally strong on all length scales, including length scales $l_{d}>l_{m}$ and may have effects similar to disorder clusters with a linear extent $l_{d}$. The disappearance of the first order behavior in our simulation may thus be qualitatively comparable to the disappearance of the first order behavior observed in experiments at fields $B>B_{u c}$.

\section{B. Effects on order parameter correlations}

By investigation of the order parameter correlations in the layered system near and below values of $\alpha_{T}$ for which a first order transition occurs in the clean system, we may gain some qualitative information about the low temperature vortex state where disorder has destroyed the transition, i.e. for $B_{l c, \text { clean }}<B<B_{l c}$, $\left(B_{l c \text {,clean }}\right.$ is the value of $B_{l c}$ in the clean limit) or for $B>B_{u c}$. We choose to investigate the disordered system for a low coupling value, $\left|\alpha_{2 T} \eta\right|=0.05$, for which we see very large discontinuities at the transition in the clean system (see Fig. 9 and 11). 

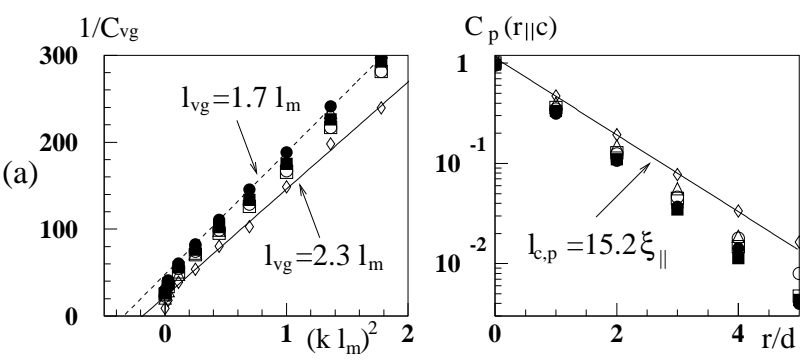

(c)

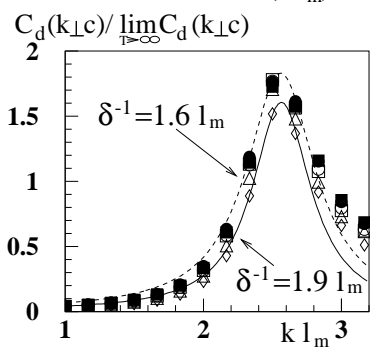

$\mathrm{C}_{\mathrm{d}}\left(\mathrm{k}=\mathrm{G}, \mathrm{r}_{\|} \mathrm{c}\right) / \lim _{T \rightarrow \infty} \mathrm{C}_{\mathrm{d}}(\mathrm{k}=\mathrm{G}, \mathrm{r}=0)$

(e)

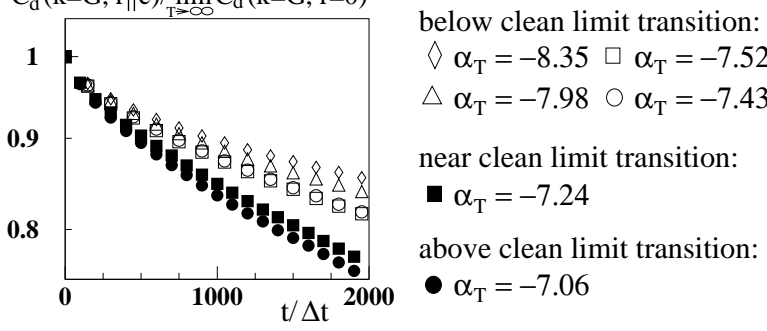

FIG. 13. Plots of the order parameter correlations with disorder for $\left|\alpha_{2 T} \eta\right|=0.05, D=0.72, N_{a b}=72, N_{c}=30$ : (a) vortex glass correlator, (b) phase correlations along the $c$-axis, (c) structure factor, (d) density correlations along the $c$-axis, (e) slowest time decay. All solid line fits are for $\alpha_{T}=-8.35$, and the corresponding correlation lengths are indicated. In (a) and (c) the dashed lines are fits for $\alpha_{T}=-7.06$.

To probe if the system is in a vortex glass state we measure the vortex glass correlator [5] defined as the disorder average over

$$
C_{v g}\left(\mathbf{r}^{\prime}\right)=\frac{\left\langle\psi^{*}(\mathbf{r}) \psi\left(\mathbf{r}+\mathbf{r}^{\prime}\right)\right\rangle\left\langle\psi(\mathbf{r}) \psi^{*}\left(\mathbf{r}+\mathbf{r}^{\prime}\right)\right\rangle}{Q^{4}},
$$

where $Q$ is defined in Sec. [1]. $C_{v g}$ is a measure of the phase correlations in the system. We can determine the Fourier transform of this correlator from averages over the LLL coefficients as described in the Appendix, Sec. B 1. $C_{v g}$ is in a clean system trivially short-ranged. It is gauge dependent but has typically a Gaussian decay behavior, $\ln \left(C_{v g}\right) \sim-r^{2}$ [48, which can for $D=0$ be observed in our simulation. In the presence of disorder, decay with a finite, temperature dependent length scale is expected for a disordered vortex liquid [5]. Exponential decay with a correlation length $l_{v g}$ in real space would appear as

$$
C_{v g}(k) \propto \frac{1}{k^{2}+l_{v g}^{-2}} .
$$

In Fig. 13 we show measurements of different density and phase correlators at a disorder strength for which all signs of a first order phase transition have disappeared from the heating and cooling measurements (not shown). In Fig. 13(a) $1 / C_{v g}$ is plotted against $k^{2}$. The behavior is in the plotted regime linear for $\left(k l_{m}\right)^{2}>0.25$. The data for wave vectors $\left(k l_{m}\right)^{2}<0.25$ corresponds to correlations more than a third of the circumference of the sphere. It is strongly affected by finite size effects and is therefore not taken into account for linear fits according to Eq. 14. The linear fits in Fig. 13(a) have rather large errors. Firstly insufficient averaging over disorder configurations causes statistical noise. Secondly and more importantly the intercept $-l_{v g}^{-2}$ depends sensitively on the exact choice of the linear fitting regime. It is however visible that for any choice it is finite and decreases with increasing $\left|\alpha_{T}\right|$. Although $l_{v g}$ grows with decreasing temperature, it is in the measured temperature regime only of the order of a lattice spacing. No long-range phase correlations typical for a vortex glass [5] are visible.

The correlators we plot in Fig. 13(b), (c), (d) and (e) have been introduced in Sec. IV. In Fig. 13 filled symbols correspond to temperatures just above and at the location of the first order transition in the clean system and empty symbols to temperatures below it. In none of the static correlators is there any rapid change of behavior across the location of the first order transition in the clean limit. However, in the relaxation behavior in Fig. 13(e) an accelerated slowing down reminiscent of the discontinuity in the clean limit is still visible.

All correlation lengths increase with $\left|\alpha_{T}\right|$. The correlation lengths $l_{a b}=2 \delta^{-1} l_{m}$ and $l_{c}$ from density as well as from phase correlations are reduced, for low temperatures by up to an order of magnitude, compared to the clean system (see Fig. 9). Unlike in the clean system, $l_{c, p}$, i.e. the decay length of phase correlations along the $c$-axis, is distinctly larger than $l_{c, d}$, the density-density correlation length. The phase correlation length in the plane from a fit of the vortex glass correlator at $\alpha_{T}=-8.35$ is of the same order as the density-density correlation length at the same temperature. Although $\alpha_{T}=-8.35$ is distinctly below the phase boundary in the clean system, no signs of long-range phase correlations expected for a vortex glass phase are visible.

We have not attempted a quantitative analysis of relaxation behavior in the layered system plotted in Fig. 13. (e), because we have not monitored the decay behavior over long enough time time intervals to see the dominant decay rates. However, we expect a behavior which is qualitatively similar to the the 2D limit of the disordered vortex liquid. We give in the following an analysis of this relaxation behavior, which extends and partly supersedes our earlier analysis given in Ref. [35]. We include with Fig. 16 a quantitative correction to data from Ref. 35] which was affected by an error in the simulation code. 


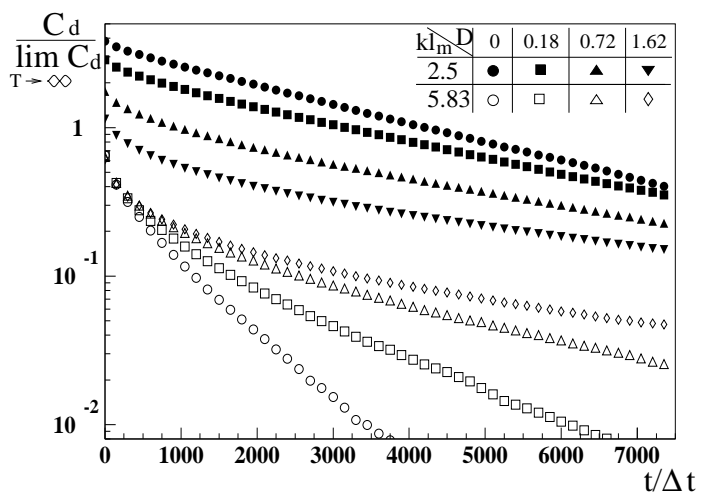

FIG. 14. Typical relaxation behavior with increasing disorder at $k l_{m}=2.5$ (near the first reciprocal lattice vector) and for an arbitrary different value of $k . N_{a b}=72$ and $\alpha_{2 T}=-9$.

Examples of the relaxation behavior of $C_{d}(k, t)$ with and without disorder are shown in Fig. 14 for the 2D limit. The relaxation behavior in samples with disorder is richer than our earlier analysis 35 suggests. A seemingly faster decay than in the clean sample is visible at short times. However, this faster decay characterises only the initial decay behavior, which becomes clear in the data in Fig. 14 for the case of strong disorder. At late times slow exponential decay on a time scale $\tau_{f}$ due to pinning to the disorder becomes visible. To distinguish the two regimes of decay, we define an initial relaxation time $\tau_{\text {in }}$ by $\tau_{\text {in }}=\tau_{1 / 2} / \ln (2)$, where $\tau_{1 / 2}$ marks the time when the correlator has decayed to half its initial value. The final time scale $\tau_{f}$ can be found by exponential fits to the decay at late times.

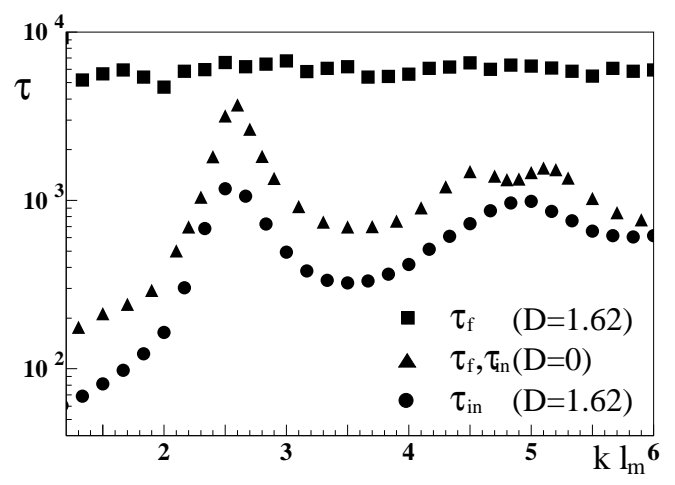

FIG. 15. Initial and final relaxation time scales of the decay of the density-density correlator in reciprocal space with and without disorder. For $D=0$ system size $N_{a b}=200$, for $D=1.62 N_{a b}=72 . \alpha_{2 T}=-9$.

Figure 15 shows a typical logarithmic plot of the $k$ dependence of initial and final relaxation times in the case of disorder compared to the clean case at the same temperature. The initial relaxation times $\tau_{i n}$ in the disordered case show qualitatively the same $k$ dependence re- flecting the hexagonal structure as in clean samples [35], while the final relaxation times $\tau_{f}$ do not depend on $k$. This seems natural, because the disorder contributions are on average equally strong for all $k$.
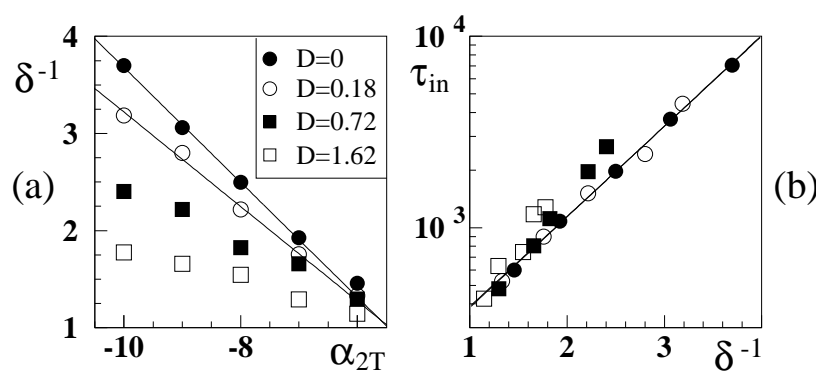

FIG. 16. (a) The inverse width of the first peak in the structure factor with linear fits for no and weak disorder in the low temperature regime. For $\alpha_{2 T}=-10$ and $D=0$ $N_{a b}=200$, otherwise $N_{a b}=72$. (b) Data of length and time scales plotted as $\ln \left(\tau_{i n}\right)$ versus $\delta^{-1}$. With linear fits for $D=0$ and $D=0.18$.

The dependence of the crystalline correlation length on $\alpha_{2 T}$ is plotted for different disorder strengths in Fig. 16.(a) and the dependence of the initial relaxation times on this length in Fig. 16(b). The initial relaxation times in the disordered system follow like in the clean system activated dynamics [35, in which the dominant relaxation time scale grows linearly with the crystalline correlation length.

The relaxation behavior in the disordered layered system plotted in Fig. 13 looks similar to the 2D behavior at early times; the initial relaxation is faster than in the clean case. A more quantitative analysis of the relaxation behavior in layered systems would be of some interest in comparison to resistivity data in disordered samples. An analysis of the resistive behavior in YBCO before and after artificial introduction of point defects by electron irradiation 49] concluded that transport in the disordered vortex liquid is dominated by viscosity rather than single vortex pinning, i.e. that the equilibrium relaxation time scales in simulations without a driving field could be relevant time scales. In the resistive measurements after irradiation in Ref. [4] no signs of a continuous vortex glass transition replacing the first order behavior before irradiation have been found. This absence of any thermodynamic phase transition in the case of strong disorder is in agreement with our simulation results.

\section{DISCUSSION AND SUMMARY}

In our numerical work we have found a first order transition which on the one hand coincides with the first order transition in $\mathrm{YBCO}$, but on the other hand is not associated with vortex lattice melting. An analysis of 
what triggers the decoupling transition at the particular $\alpha_{T}$ where it is observed is beyond the scope of this paper. The answer to this question could lie in the observation made by Pierson and Valls [37] that the first order transition in YBCO coincides with the onset of 3D LLL fluctuations. Because even strongly layered systems are $3 \mathrm{D}$ in nature below the first order transition, the onset of 3D LLL fluctuations can be expected to occur at a fairly constant value of $\alpha_{T}$, and this may be reflected in approximately constant $\alpha_{T}$ along the phase transition line.

Some readers may believe that the nature of the first order transition in YBCO is vortex lattice melting and dismiss the absence of a vortex lattice state below the transition in our simulation as an artifact of the spherical boundary conditions we use. In this case however it would seem astonishing that this first order transition associated with vortex lattice melting should quite happily persist in our simulation in the absence of a vortex lattice. Besides, a first order vortex decoupling transition [27] and another similar [50] thermodynamic transition [24], both not associated with vortex lattice melting, have also been observed in simulations of different models using periodic boundary conditions.

\section{A. Comparison with previous LLL-LD simulations}

From previous simulations using the same model, but with periodic boundary conditions, a single vortex lattice melting transition is reported [29]30]. We find there is a disagreement in the location of the transition between our simulation and Ref. 30 for weak couplings. This is certainly due to the different choice of boundary conditions, because in the the $2 \mathrm{D}$ case the two choices of boundary conditions are known to yield different results [32,36, 51. As the coupling increases, the disagreement in the location of the transition between our results and those from Ref. [30] disappears. For couplings high enough to see the critical end-point, the LLL-LD model has never been investigated using periodic boundary conditions.

The LLL-LD model simulations using periodic boundary conditions exhibit a vortex lattice state below the transition. If the transition was of the same nature for periodic boundary conditions as for spherical layers, the apparent vortex lattice state could be accounted for by the use of system sizes smaller than the correlation lengths. (The largest system sizes in these simulations were of the order of only 40 vortices $\times 20$ layers.) Small system sizes together with the restrictions in degrees of freedom imposed by periodic boundary conditions may make a vortex liquid with very long length scales indistinguishable from a vortex lattice.

\section{B. Relevance for BSCCO}

We have so far only compared our numerical data with experiments in YBCO. Near the phase transition in BSCCO neither the LLL approximation nor the LD model are valid. The phase diagram of BSCCO has a first order transition line [52], which occurs at much smaller applied magnetic fields than in YBCO. Near the BSCCO transition $H / H_{c 2}$ is of the order $10^{-3}$. This means that the LLL approximation we use in our simulation cannot be expected to apply. The strongly anisotropic character of BSCCO is such that Josephson coupling between the layers may be negligible compared to electromagnetic coupling effects [53] and so BSCCO is not accurately described by the LD model.

However, some qualitative points of comparison can be made. It is for example noteworthy that in BSCCO the material parameters $\kappa$ as well as the layer periodicity $d$, $T_{c}$ and $\partial B_{c 2} / \partial T$ are of the same order as in YBCO, but typical estimates of the mass anisotropy $\gamma$ are between one and two orders of magnitude larger. This means that the end-point of our numerical transition line translates via the relation $B_{l c} \propto 1 / \gamma^{4}$ to fields that are between 4 and 8 orders of magnitude lower than in YBCO. An experimental observation of a lower critical end-point is therefore not to be expected in BSCCO.

In the low temperature limit, where all length scales are large, both YBCO and BSCCO should show the same universal behavior. Should our phase diagram be valid so that there is no thermodynamic vortex lattice melting transition at a finite temperature below the experimentally observed first order transition in YBCO, then BSCCO should also be in a vortex liquid state below the first order transition. The consequence that the first order transition in BSCCO is not of a genuine vortex lattice melting character is in agreement with recent experimental evidence that hexagonal neutron diffraction patterns, which signify that a vortex lattice or a vortex liquid with very long length scales, can be observed above as well as below the first order transition line 54 .

\section{Summary}

We have numerically calculated the phase diagram of a layered superconductor and found a first order transition line between two vortex liquid states. The length scales of order parameter correlations parallel and perpendicular to the magnetic field as well as the longest relaxation time scales increase discontinuously at the transition but remain finite as the temperature is lowered. As the coupling strength between layers increases, the discontinuities in length and time scales decrease until the transition line ends at a critical end-point. Shape, location and anisotropic scaling properties of the transition line and 
its end-point as well as the size of the reversible magnetization discontinuity are in excellent agreement with the experimental first order transition line and its low field end-point in YBCO. The approximate location of the end-point can be predicted from a qualitative argument assuming that the transition is of a layer decoupling nature. However, the exact quantitative agreement of the location of the end-point with experiments by Schilling et al. [10] could be a chance result due to cancellation of inaccuracies of our numerical model at low fields and/or finite size effects with disorder effects in real samples.

Our results suggest that the transition in YBCO, which is commonly interpreted as vortex lattice melting, is of a liquid-liquid nature, with a low temperature vortex liquid phase in which length scales grow exponentially fast and time scales due to activated dynamics doubly exponentially fast with decreasing temperature. We argue that because not far below the first order transition the vortex liquid is highly viscous and effectively crystalline over large length scales, our picture can account for many experimental features including resistance drops and Bragg peaks, which have so far been taken as evidence for the vortex lattice melting scenario.

We have investigated the effect of quenched random point disorder on the system and found that the first order transition can be suppressed by strong disorder and that the low field critical end-point can be shifted to higher fields by weak disorder. We find no evidence of a thermodynamic transition to a Bragg glass phase or vortex glass phase replacing the first order transition line in the presence of strong disorder.

\section{ACKNOWLEDGEMENTS}

We would like to thank Sai-Kong Chin and Matthew Dodgson for useful interactions. AKK acknowledges financial support from a Manchester University Research Studentship and EPSRC.

\section{APPENDIX A: DETAILS OF THE HAMILTONIAN}

\section{Quartic energy term}

The quartic coupling term in Eq. 3 can in each layer be expressed in terms of the LLL eigenfunctions in the following way (we omit the layer numbering indices):

$$
\frac{\mathcal{H}_{\text {quartic }}}{k_{B} T}=\frac{1}{k_{B} T} \frac{\beta_{2 D}}{2} d_{0} \int d^{2} r|\psi|^{4}=\frac{1}{2 N} \sum_{p=0}^{2 N}\left|U_{p}\right|^{2},
$$

with $U_{p}=\sum_{m=\max (0, p-N)}^{\min (p, N)} f(m, p-m) v_{m} v_{p-m}$, with $f(m, n)=A_{m} A_{n}(B(m+n+1,2 N-m-n+1))^{1 / 2}$, where $A_{n}$ as defined in Eq. 1 and $B$ is the beta function.

\section{Coupling to disorder}

Let the Gaussian random disorder $\Theta(\mathbf{r})$ in each layer be expanded as

$$
\Theta=\frac{k_{B} T}{d_{0} Q^{2}} \sum_{l=0}^{\infty} \sum_{m=-l}^{l} a_{l}^{m} \tilde{Y}_{l}^{m},
$$

where the $\tilde{Y}_{l}^{m}$ are spherical harmonics normalized with respect to the spherical surface of radius $\sqrt{N / 2}$. For real $\Theta, a_{l}^{m *}=a_{l}^{-m}$. The $a_{l}^{m}$ are chosen independently from a random Gaussian distribution with zero mean and variance $\overline{\left|a_{l}^{m}\right|^{2}}=D$. Calculating the disorder correlations $\overline{\Theta(\mathbf{r}) \Theta\left(\mathbf{r}^{\prime}\right)}$ and inserting $\Delta$ as defined in Eq. 目 fixes $D=\left(d_{0} Q^{2} / k_{B} T\right)^{2} \Delta$. For the integration of (layer indices omitted)

$$
\begin{aligned}
\frac{\mathcal{H}_{d i s}}{k_{B} T} & =\frac{d_{0}}{k_{B} T} \int d^{2} r \Theta(\mathbf{r})|\psi(\mathbf{r})|^{2} \\
& =\sum_{p, q=0}^{N} \sum_{l=0}^{\infty} \sum_{m=-l}^{l} \int d^{2} r a_{l}^{m} \tilde{Y}_{l}^{m} v_{p}^{*} \phi_{p}^{*} v_{q} \phi_{q} .
\end{aligned}
$$

we define for $0 \leq m \leq l$ and $0 \leq q \leq N-m$

$$
\begin{aligned}
& I_{l, q}^{m}=\int d^{2} r \tilde{Y}^{m} \phi_{q+m}^{*} \phi_{q} \\
& =A_{q} A_{q+m} \sqrt{\frac{(2 l+1)(l+m) !}{2 \pi N(l-m) !}} \frac{(-1)^{m}}{m !} B(N-q+1, q+m+1) \\
& \quad \times{ }_{3} F_{2}(m-l, m+l+1, q+m+1 ; \quad m+1, N+m+2 ; 1),
\end{aligned}
$$

where ${ }_{3} F_{2}$ is a generalized hypergeometric function. Substituting these integrals into Eq. A2, truncating the contributions of noise components with frequencies $l>$ $l_{\max }=N$ (for a standard system size of $N=72$ these are more than $10^{20}$ times smaller than the largest contributing terms), and defining

$$
g_{q, p}=\sum_{l=p-q}^{N} a_{l}^{p-q} I_{l, q}^{p-q}
$$

yields

$$
\frac{\mathcal{H}_{\text {dis }}}{k_{B} T}=\sum_{q=0}^{N} \sum_{p=q}^{N} g_{q, p} v_{p}^{*} v_{q}+\delta_{q, p} \times \text { c.c. } .
$$

Note that once $g_{q, p}$ is calculated the original coefficients $a_{l}^{m}$ can be discarded and need therefore not all simultaneously be held in memory. 


\section{APPENDIX B: CORRELATIONS}

To compute correlations in reciprocal space, we perform the spherical equivalent of a Fourier transform, the expansion in the discrete set of normalized spherical harmonics, $\tilde{Y}_{l}^{m}(\mathbf{r})$. To a value of $l$ corresponds $k=l / R$. Because the liquid is isotropic, the correlator in $k$ space depends only on the magnitude of $k$, i.e. only on $l$ and not on $m$. For better averaging we calculate the correlator for all $m$ and average over the different $m$.

\section{Vortex glass correlations}

We calculate the Fourier transform of the correlator as defined in Eq. 13 in terms of thermal averages of the LLL coefficients.

$$
\begin{aligned}
& C_{v g}(l / R)=\frac{1}{Q^{4}(2 l+1) 4 \pi R^{2}} \int d^{2} r \int d^{2} r^{\prime} \\
& \sum_{m=-l}^{l} \tilde{Y}_{l}^{m}(\mathbf{r}) \tilde{Y}_{l}^{-m}\left(\mathbf{r}^{\prime}\right)\left\langle\psi^{*}(\mathbf{r}) \psi\left(\mathbf{r}^{\prime}\right)\right\rangle\left\langle\psi(\mathbf{r}) \psi^{*}\left(\mathbf{r}^{\prime}\right)\right\rangle \\
& =\sum_{m=-l}^{l} \sum_{n, n^{\prime}=\max (0,-m)}^{\min (N, N-m)} \frac{I_{l, n}^{|m|} I_{l, n^{\prime}}^{|m|}\left\langle v_{n+m}^{*} v_{n^{\prime}+m}\right\rangle\left\langle v_{n} v_{n^{\prime}}^{*}\right\rangle}{2 \pi N(2 l+1)},
\end{aligned}
$$

where the $I_{l, n}^{m}$ are defined in Eq. A3.

\section{Density-density correlations along the $c$-axis}

The real space density-density correlator for $\Delta t=0$ and $\Delta \mathbf{r} \| \hat{c}, \Delta r=n d$ is given by

$$
C_{d}(n d)=\frac{\left\langle|\psi(\mathbf{r})|^{2}|\psi(\mathbf{r}+n d \hat{c})|^{2}\right\rangle}{\left\langle|\psi|^{2}\right\rangle^{2}}-1 .
$$

Taking the spatial average over all $\mathbf{r}$ in one layer involves the same integrals over LLL eigenfunctions as the calculation of the quartic energy term. Together with spatial averaging over different layers this yields

$$
C_{d}(n d)=\frac{\sum_{p=1}^{N_{c}}\left\langle\sum_{q=0}^{2 N_{a b}} \tilde{U}_{p, q}^{n *} \tilde{U}_{p, q}^{n}\right\rangle}{\sum_{p=1}^{N_{c}}\left\langle\sum_{q=0}^{N_{a b}} v_{p, q}^{*} v_{p, q}\right\rangle^{2}}-1,
$$

where

$$
\tilde{U}_{p, q}^{n}=\sum_{m=\max \left(0, q-N_{a b}\right)}^{\min \left(q, N_{a b}\right)} f(m, q-m) v_{p, m} v_{p+n, q-m},
$$

with $f(m, q-m)$ as defined in Sec. A 1 .

The $2 \mathrm{D}$ density-density correlator in $\mathrm{k}$ space from reference [32] is easily generalized to three dimensions as

$$
\begin{aligned}
& C_{d}(l / R, n d, \Delta t)=\frac{2 \pi N_{a b}}{(2 l+1)} \sum_{p=1}^{N_{c}}\left\langle\sum_{q=0}^{N_{a b}} v_{p, q}^{*} v_{p, q}\right\rangle^{-2} \times \\
& \sum_{m=-l}^{l} \sum_{p=1}^{N_{c}}\left\langle\sum_{q=\max (0,-m)}^{\min \left(N_{a b}, N_{a b}-m\right)} v_{p, q+m}^{*}(t) v_{p, q}(t) I_{l, q}^{|m|} \times\right. \\
& \left.\sum_{q^{\prime}=\max (0,-m)} v_{p+n, q^{\prime}+m}\left(t^{\prime}\right) v_{p+n, q^{\prime}}^{*}\left(t^{\prime}\right) I_{l, q^{\prime}}^{|m|}\right\rangle_{c},
\end{aligned}
$$

where $c$ signifies the connected average and the $I_{l, n}^{m}$ are defined in Eq. A3. The high temperature limit of this correlator is for $t=n=0$ easily calculated analytically [32] as

$$
\lim _{\alpha_{2 T} \rightarrow \infty} C_{d}(l / R, 0,0)=\frac{(N !)^{2}}{(N-l) !(N+l+1) !} .
$$

For analysis of relaxation times this correlator is numerically Fourier transformed in the $c$-direction. To make $C_{d}(l / R, n d, t)$ converge in the continuum limit we choose $\xi_{\|}$as unit of length when integrating along the $c$-axis:

$$
C_{d}(l / R, q, t)=\frac{1}{\sqrt{\eta}} \sum_{n=1}^{N_{c}} C_{d}(l / R, n d, t) \cos (q \times n d)
$$

where q takes values $q=2 \pi m /\left(N_{c} d\right)$ for $m=0 \ldots N_{c} / 2$.

\section{Phase correlations along the $c$-axis}

The phase correlations along the $c$-axis as defined in Eq. 12 are easily expressed in terms of the LLL coefficients using the orthonormality of the LLL functions and the identity $\alpha_{T}=2 \pi \alpha_{H} / \beta Q^{2}$ :

$$
\begin{aligned}
C_{p}(n d) & =\frac{\alpha_{T} \beta}{2 \pi \alpha_{H}}\left\langle\frac{1}{4 \pi R^{2}} \int d^{2} r \psi^{*}(r) \psi(r+n d)\right\rangle \\
& =\frac{\alpha_{T}}{\alpha_{2 T}} \times \frac{1}{2 \pi N_{a b} N_{c}}\left\langle\sum_{p=0}^{N_{a b}} \sum_{q=1}^{N_{c}} v_{p, q}^{*} v_{p, q+n}\right\rangle .
\end{aligned}
$$

\section{APPENDIX C: FINITE SIZE EFFECTS}

The limitations in the region of parameter space for which we can run our simulation as well as the limitations in accuracy of our measurements are mainly due to limited availability of processor time. The simulation time grows, as seen in Sec. IVB, roughly doubly exponentially fast with decreasing $\alpha_{T}$. The ratio of cpu time to simulation time, given essentially by the number of floating point operations needed for one update of the state, depends linearly on the number of layers $N_{c}$, but due to the quartic energy term for large systems 
quadratically on the number of vortices $N_{a b}$. Finite size effects become important and therefore large system sizes necessary when correlation lengths grow large, which is unfortunately just in the regime of phase space where relaxation times also grow large, namely at low $\alpha_{T}$.

\section{Effects of limited $N_{a b}$}

Most of our data have been taken using $N_{a b}=72$. For high temperatures finite size effects are negligible, because there is little in-plane order and the range of crystalline correlations in any one layer is much smaller than the spherical dimensions. The topological disorder due to the 12 topological defects imposed by spherical geometry 32] becomes at high temperatures negligible against the strong thermal disorder in the system. With decreasing $\alpha_{T}$ and growing $l_{a b}$, finite size effects due to finite $N_{a b}$ become important. For any one $\alpha_{T}$, the effects of the limited number of vortices are the more severe the lower the coupling strength $\left|\alpha_{2 T} \eta\right|$ and the $2 \mathrm{D}$ effective temperature $\alpha_{2 T}$ are, because outside the continuum limit inplane order increases with decreasing coupling strength, indicated by a smaller correlation length $l_{a b}$ (see Fig. 9) and smaller $\beta_{A}$ (see Fig. 3). As a consequence of too small $N_{a b}$, we see a decrease in length scales of crystalline correlations as previously observed in 2D systems [32] as well as a decrease of correlations along the $c$-axis. We interpret agreement of measurements taken with $N_{a b}=72$ and $N_{a b}=144$ as indication that with $N_{a b}=72$ we are already close to the thermodynamic limit.

\section{Effects of limited $N_{c}$}

Finite size effects due to limited numbers of layers are for equilibrium measurements not a severe problem, because an increase of $N_{c}$ increases the cpu time only linearly, and improves the rate of thermal averaging by the same amount. Therefore the disadvantage of increasing $N_{c}$ is only the increase in equilibration time as long as enough memory is available. If $N_{c} / 2$, the distance over which correlations decay, is not distinctly larger than the correlation length along the layers (see Sec. IV], we find that correlations both parallel and perpendicular to the $c$-axis are artificially enhanced and slower than exponential decay behavior is observed, as visible in Fig. 17 for $N_{c}=30$. For equilibrium measurements of length and time scales, we always have $N_{c}>10 \times l_{c} / d$, which required on occasion using $N_{c}$ up to 300 layers with 144 vortices per layer.

\section{Thermal averaging and initial relaxation}

Initial relaxation has proved a very difficult problem in our 3D simulations, where relaxation times are so long that the entire simulation time is often limited to only few times the longest relaxation time. In principle, the relaxation can be started from an arbitrary state, where obvious choices are a random state or a ground state. The relaxation from a ground state has the disadvantage that it is very slow for low $\alpha_{T}$. On the other hand it is fairly easy to judge how far the system has relaxed when started with the same state in every layer. Because our system is always in a liquid state, a slower than exponential decay of correlations in the $c$-direction (which is in a sufficiently large system always removable by further equilibration) is a reliable sign of insufficient equilibration. A case of insufficient relaxation can be seen in Fig. 17 for $N_{c}=180$. When using systems started from a ground state, they are always allowed to relax for longer than the longest relaxation time $\tau$, in most cases longer than $5 \tau$. Relaxation from a random state has got the advantage of being faster than from a ground state. However, we found measurements from an insufficiently equilibrated random state often indistinguishable from equilibrium measurements at a higher temperature, and therefore avoided starting equilibrium measurements from a random state.

The most efficient way to obtain a well equilibrated system is to cool down or heat up a configuration obtained for a similar temperature and coupling strength and identical system size. We used this method whenever such configurations were available. The cooling or heating rates in these cases are similar to those used in our hysteresis measurements.

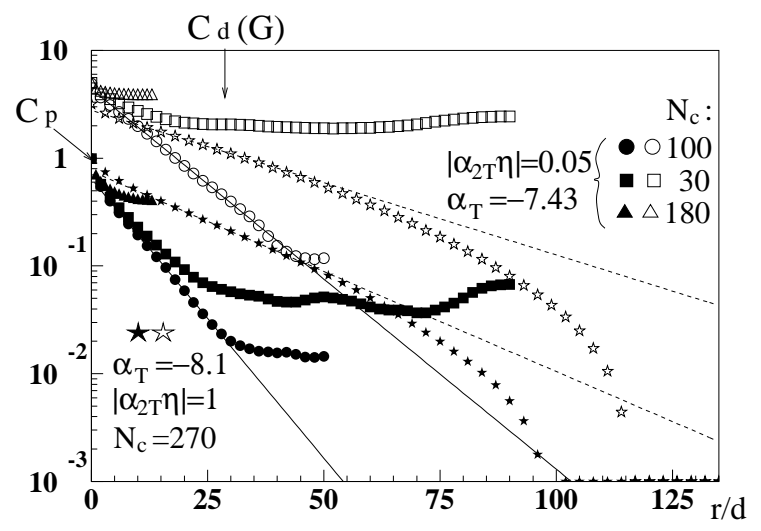

FIG. 17. Typical measurements of static correlations along the $c$-axis. Open symbols are density correlations, filled symbols are phase correlations. Note for squares that correlations are enhanced by insufficient system size and for triangles the incomplete relaxation of the system. Statistical noise can for large $r$ suggest slower than exponential decay (circles) as well as faster than exponential decay (stars). For all systems $N_{a b}=72$. 


\section{Loss of first order behavior}

The change of the hysteresis at the first order transition for decreasing numbers of layers can be seen in Fig. 18. The correlation length along the $c$-axis (see Sec. IV] just above the transition is for $\left|\alpha_{2 T} \eta\right|=0.4$ approximately 6 layers. For $N_{c}=20$, correlations along the $c$-axis are enhanced significantly just above the transition. The system does not remain in the high entropy, decoupled state, and the hysteresis is lost. This effect is visible in Fig. 18 in the reduction of the layer independence parameter $\Gamma$ (defined in Sec. IIIB) just above the transition in case of the smaller system size. The loss of hysteresis with decreasing system size is rather sudden. Further increase in system size beyond 5-6 times the range of $c$-axis correlations affects the hysteresis measurements very little.

In all cases the system sizes used for the hysteresis measurements are more than five times the correlation length in $c$-direction just above the transition. The correlation lengths are for selected values of $\left|\alpha_{2 T} \eta\right|$ known from equilibrium measurements in large systems and otherwise estimated by interpolation. We are aware that the system sizes used for the sweep measurements are in most cases not distinctly larger than the range of correlations below the transition. However, the observed size of the jumps does not seem much affected by this; the discontinuities in the order parameter magnitude seen in equilibrium measurements in much larger systems for selected $\left|\alpha_{2 T} \eta\right|$ agree well with the results from sweep measurements.

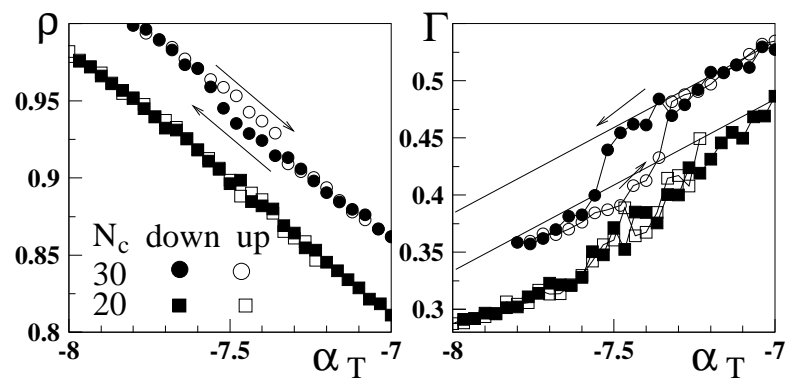

FIG. 18. Order parameter density $\rho$ and the measure of layer independence $\Gamma$ upon heating and cooling. For insufficient number of layers, $\Gamma$ is reduced on the high temperature side of the transition and the first order behavior is lost. For $N_{c}=20 \rho$ and $\Gamma$ are offset by -0.05 (equal levels marked by solid lines). $N_{a b}=72$ and $\left|\alpha_{2 T} \eta\right|=0.4$.

A very important point to verify is that the loss of hysteresis at the critical point is not an effect of insufficient numbers of layers. We have made sure that the system sizes used near the critical point are not only more than five times the correlation length, but also that the loss of the transition at $\left|\alpha_{2 T} \eta\right|=2.5$ occurs in a system that is not only in terms of layers, but also in terms of the natural length scale $\xi_{\|}$, larger than the system for $\left|\alpha_{2 T} \eta\right|=2$, where a transition is still visible. For $\left|\alpha_{2 T} \eta\right|=2$ and $N_{c} d / \xi_{\|}=65$ we see a clear transition, while for $\left|\alpha_{2 T} \eta\right|=2.5$ and $N_{c} d / \xi_{\|}=80$ there is no sign of a transition in the range $-8.3<\alpha_{T}<-7.3$.
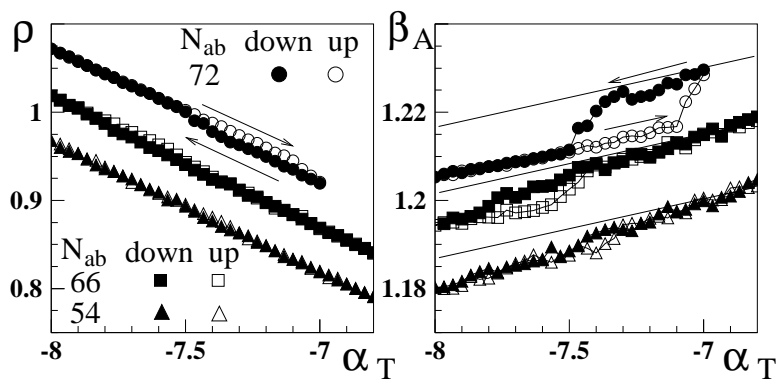

FIG. 19. Order parameter density $\rho$ and Abrikosov number $\beta_{A}$ upon heating and cooling. For insufficient number of vortices per layer, $\beta_{A}$ is increased on the low temperature side of the transition and the first order behavior is lost. For $N_{a b}=66$ and $N_{a b}=54, \rho$ is offset by -0.05 and -0.1 respectively and $\beta_{A}$ is offset by -0.015 and -0.03 respectively (equal levels marked by solid lines). $N_{c}=12$ and $\left|\alpha_{2 T} \eta\right|=0.05$.

If the number of vortices per layer $N_{a b}$ is reduced, the hysteresis at the transition first decreases and then disappears. For the general reasons outlined in Sec. C 1 the effect becomes stronger as $\left|\alpha_{2 T} \eta\right|$ decreases. Below some critical $N_{a b}$, which increases with decreasing $\left|\alpha_{2 T} \eta\right|$, the in-plane order below the transition, reflected in the Abrikosov Ratio $\beta_{A}$, is so much affected by the spherical topology that the discontinuity in $\beta_{A}$ vanishes and the transition disappears. This behavior is shown in Fig. 19 for $\left|\alpha_{2 T} \eta\right|=0.05$. The size of the discontinuities decreases noticeably between $N_{a b}=72$ and $N_{a b}=66$. For $N_{a b}=54$ the transition has disappeared. For $N_{a b}=72$, we still measure a transition at $\left|\alpha_{2 T} \eta\right|=0.02$, but not at $\left|\alpha_{2 T} \eta\right|=0.01$. Thus the need of increasing $N_{a b}$ limits the exploration of the phase diagram for very low $\left|\alpha_{2 T} \eta\right|$.

We estimate that the limitation of the number of vortices we study to $N_{a b}=72$ affects our hysteresis measurements for $\left|\alpha_{2 T} \eta\right| \leq 0.05$. We can detect size dependence in the location of the phase transition for system sizes $N_{a b}=72$ and $N_{a b}=144$ only for $\left|\alpha_{2 T} \eta\right|<0.05$ (see the phase diagram in Fig. 4). The hysteresis measurements plotted in the same figure show that the size of the discontinuities at the phase transition does not change noticeably between $N_{a b}=72$ and $N_{a b}=144$ for $\left|\alpha_{2 T} \eta\right|=0.14$. For $\left|\alpha_{2 T} \eta\right| \gg 0.14$, which applies to the region near the critical point, the system should at the phase transition be well simulated using $N_{a b}=72$. This is confirmed by the agreement in the magnetization discontinuity between $N_{a b}=72$ and $N_{a b}=144$ in Fig. 2(b) for $T=89.3$. 
[1] A. Fetter and P. Hohenberg, in Superconductivity, edited by R. Parks (Dekker, New York, 1969), Vol. 2.

[2] G. J. Ruggeri and D. J. Thouless, J. Phys. F 6, 2063 (1976).

[3] G. Blatter et al., Rev. Mod. Phys. 66, 1125 (1994).

[4] L. I. Glazman and A. E. Koshelev, Phys. Rev. B 43, 2835 (1991); L. L. Daemen et al., Phys. Rev. Lett. 70, 1176 (1993).

[5] D. S. Fisher, M. P. A. Fisher and D. A. Huse, Phys. Rev. B 43, 130 (1991).

[6] T. Giamarchi and P. Le Doussal, Phys. Rev. Lett. 72, 1530 (1994); Phys. Rev. B 55, 6577 (1997).

[7] W. K. Kwok et al., Phys. Rev. Lett. 72, 1092 (1994).

[8] U. Welp et al., Phys. Rev. Lett. 76, 4809 (1996).

[9] R. Liang, D. A. Bonn and W. N. Hardy, Phys. Rev. Lett. 76, 853 (1996).

[10] A. Schilling et al., Nature 382, 791 (1996).

[11] A. Junod, et al., Physica C 275, 245 (1997).

[12] M. Roulin et al., Phys. Rev. Lett. 80, 1722 (1998).

[13] A. Schilling et al., Phys. Rev. Lett. 78, 4833 (1997).

[14] G. Blatter, V. G. Geshkenbein and A. I. Larkin, Phys. Rev. Lett. 68, 875 (1992).

[15] M. Roulin, A. Junod and E. Walker, Physica C 296, 137 (1998).

[16] G. W. Crabtree et al., J. Low Temp. Phys. 105, 1073 (1996).

[17] K. Deligiannis et al., Phys. Rev. Lett. 79, 2121 (1997).

[18] H. Safar et al., Phys. Rev. B 52, 6211 (1995).

[19] P. L. Gammel et al., Phys. Rev. Lett. 59, 2592 (1987).

[20] C. M. Aegerter et al., Phys. Rev. B 57, 14511 (1998). M. T. Wylie et al., Czech. J. Phys. 46, 1569 (1996). M. Yethiraj et al., Phys. Rev. Lett. 70, 857 (1993).

[21] M. Yethiraj et al., Phys. Rev. Lett. 71, 3019 (1993).

[22] T. Chen and S. Teitel, Phys. Rev. B 55, 11766 (1997); 55, 15197, (1997); Phys. Rev. Lett. 74, 2792 (1995); S. Ryu and D. Stroud, Phys. Rev. B 57, 14476 (1998); T. J. Hagenaars et al., Phys. Rev. B 55, 11706 (1997); T. J. Hagenaars and E. H. Brandt, Phys. Rev. B 56, 11435 (1997).

[23] A. K. Nguyen and A. Sudbø, Phys. Rev. B 57, 3123 (1998); A. E. Koshelev, Phys. Rev. B 56, 11201, (1997); X. Hu, S. Miyashita and M. Tachiki, Phys. Rev. Lett. 79, 3498 (1997).

[24] A. K. Nguyen and A. Sudbø, preprint.

[25] S. A. Ktitorov, B. N. Shalaev and L. Jastrabik, Phys. Rev. B 49, 15248 (1994).

[26] H. Nordborg and G. Blatter, Phys. Rev. Lett. 79, 1925 (1997); preprint cond-mat/9803041.

[27] N. K. Wilkin and J. Jensen, Europhys. Lett. 40, 423 (1997); Phys. Rev. Lett. 79, 4254 (1997).

[28] T. Blum and M. A. Moore, in preparation.
[29] I-J. Hwang, R. Šášik and D. Stroud, Phys. Rev. B 48, 9938 (1993); R. Šášik and D. Stroud, Phys. Rev. Lett. 72, 2462 (1994); 75, 2582 (1995); Phys. Rev. B 52, 3696 (1995); 54, 12010 (1996).

[30] J. Hu and A. H. MacDonald, Phys. Rev. B 56, 2788 (1997).

[31] W. E. Lawrence and S. Doniach, in Low Temperature Physics, 12th international conference proceedings, Kyoto, Japan, edited by E. Kanda, (Keygaku, Tokyo, Japan, 1971), p.316.

[32] M. J. W. Dodgson and M. A. Moore, Phys. Rev. B 55, 3816 (1997).

[33] A. Pérez-Garrido and M. A. Moore, to be published in Phys. Rev. B.

[34] R. Ikeda, J. Phys. Soc. Jpn. 64, 1683 (1995); Z. Tešanović, A. V. Andreev, Phys. Rev. B 49, 4064 (1994).

[35] A. K. Kienappel and M. A. Moore, Phys. Rev. B 56, 8313 (1997).

[36] J. A. O'Neill and M. A. Moore, Phys. Rev. B 48, 374 (1993); H. H. Lee and M. A. Moore, Phys. Rev. B 49, 9240 (1994).

[37] S. W. Pierson and O. T. Valls, Phys. Rev. B 57, R8143 (1998).

[38] Z. Tešanović, preprint cond-mat/9801306.

[39] D. J. Thouless, Phys. Rev. Lett. 34, 946 (1975).

[40] S. P. Farrant and C. E. Gough, Phys. Rev. Lett. 34, 943 (1975).

[41] S-K. Chin and M. A. Moore, preprint cond-mat/9709347.

[42] M. A. Moore, Phys. Rev. B 45, 7336 (1992).

[43] J. Yeo and M. A. Moore, Phys. Rev. Lett. 78, 4490 (1997).

[44] D. E. Farrell et al., Phys. Rev. B 53, 11807 (1996).

[45] A. Schilling, H. R. Ott and T. Wolf, Phys. Rev. B 46, 14253 (1996).

[46] T. Ishida et al., Phys. Rev. B 56, 5128 (1997); preprint (to be published in Phys. Rev. B 58, (1998)).

[47] A. Erb et al., preprint cond-mat/9805222; J. Low Temp. Phys. 105, 1023 (1996).

[48] R. Šášik, D. Stroud and Z. Tešanović, Phys. Rev. B 51, 3042 (1995).

[49] J. A. Fendrich et al., Phys. Rev. Lett. 74, 1210 (1995).

[50] The authors of Ref. 24] do not report clear evidence for a first order transition. However, we believe that a peak in the numerical specific heat data of Ref. [24 at the transition may indicate a first order transition.

[51] J. Hu and A. H. MacDonald, Phys. Rev. Lett. 71, 432 (1993); Phys. Rev. B 49, 15263 (1994); Y. Kato and N. Nagaosa, Phys. Rev. B 47, 2932 (1992); 48, 7383 (1993).

[52] E. Zeldov et al., Nature 375, 373 (1995).

[53] G. Blatter et al., Phys. Rev. B 54, 72 (1996).

[54] E. M. Forgan et al., Czech. J. Phys. 46, 1571 (1996). 